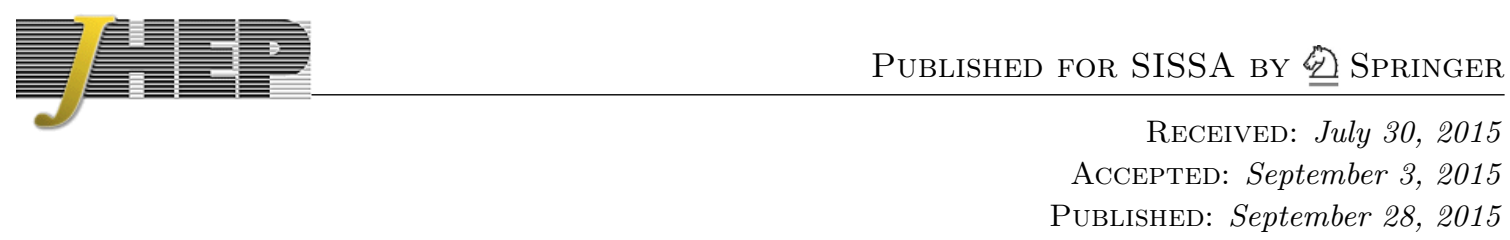

\title{
One-loop triple collinear splitting amplitudes in QCD
}

\author{
Simon Badger, Francesco Buciuni and Tiziano Peraro \\ Higgs Centre for Theoretical Physics, School of Physics and Astronomy, University of Edinburgh, \\ Edinburgh EH9 3JZ, Scotland, U.K. \\ E-mail: Simon.Badger@ed.ac.uk, Tiziano.Peraro@ed.ac.uk, \\ Francesco.Buciuni@ed.ac.uk
}

ABSTRACT: We study the factorisation properties of one-loop scattering amplitudes in the triple collinear limit and extract the universal splitting amplitudes for processes initiated by a gluon. The splitting amplitudes are derived from the analytic Higgs plus four partons amplitudes. We present compact results for primitive helicity splitting amplitudes making use of super-symmetric decompositions. The universality of the collinear factorisation is checked numerically against the full colour six parton squared matrix elements.

KEYwords: Scattering Amplitudes, Strong Coupling Expansion, QCD

ARXIV EPRINT: 1507.05070 


\section{Contents}

1 Introduction 1

2 Notation 3

3 A spinor parametrisation of the multi-collinear limit 5

3.1 Example: the tree-level MHV multi-collinear splitting amplitude $\quad 7$

3.2 One-loop basis functions for $p p \rightarrow H+2 j$ in the triple collinear limit 8

$4 \quad g \rightarrow g g g$ splitting amplitudes $\quad 8$

4.1 Colour structure and primitive amplitude decomposition 8

$\begin{array}{lll}4.2 & \text { Results } & 10\end{array}$

$5 \quad g \rightarrow \bar{q} q g$ splitting amplitudes $\quad 14$

$\begin{array}{lll}5.1 & \text { Colour structure and primitive amplitude decomposition } & 14\end{array}$

$\begin{array}{lll}5.2 \text { Results } & 15\end{array}$

6 Super-symmetric Ward identities $\quad 20$

$\begin{array}{lll}7 & \text { Cross checks } & 21\end{array}$

8 Conclusions $\quad 22$

$\begin{array}{ll}\text { A Generation of collinear phase space points } & 23\end{array}$

\section{Introduction}

A full understanding of the infrared structure of QCD matrix elements is an unavoidable step towards making precise predictions of Standard Model backgrounds at hadron colliders. In order to make finite predictions for cross-sections we must cancel infrared singularities between unresolved real radiation corrections and singularities in the virtual (loop) corrections. The study of infrared properties of perturbative gauge theories have a broader scope beyond this application since the universal behaviour provides a strong constraint on the structure of scattering amplitudes.

The soft and collinear infrared limits at next-to-leading order (NLO) have been understood long ago and general algorithms (e.g. Catani-Seymour [1] or Frixone-KunsztSigner [2]) for the computation of infrared finite cross-sections form the core of the current generation of precision tools used to make theoretical predictions for the LHC experiments.

In the last ten years or so a lot of effort has been put into generalising these techniques to next-to-next-leading order (NNLO) and a variety of different techniques now exist with 
the ability to make finite predictions for important LHC observables (e.g. references [3-8]). All of these methods rely on knowledge of the underlying factorisation properties of QCD amplitudes in the double unresolved limits at tree-level [9] and single unresolved limits at one-loop [10-14].

The first step at next-to-next-to-next-to-leading order $\left(\mathrm{N}^{3} \mathrm{LO}\right)$ has been taken recently through the complete calculation of fully inclusive Higgs production at hadron colliders up to $\mathcal{O}\left(\alpha_{s}^{5}\right)$ in the large top quark mass limit [15]. This calculation has been performed in a number of different stages building expansions around the soft limit [16-18] and using the reverse unitarity method to obtain each component of the triple-virtual [19-21], squared real-virtual [22, 23], double-virtual-real [24-27], double-real-virtual [28, 29] and triple-real radiation [30] as an expansion in the dimensional regularisation parameter. The poles of these separate contributions cancel analytically when summed together and combined with the counter-terms for UV poles [31-34] and initial state infrared singularities [35-39].

Further steps are required to extend these techniques to fully differential observables in an analogous way to the NLO and NNLO cases. Many of the infrared regions that must be accounted for in such a procedure are now fully understood. The missing ingredients that remain are the one-loop triple collinear splitting functions involving gluons. Though the factorisation of the squared matrix elements are sufficient for the construction of infrared finite cross sections, factorisation at the amplitude level [40] can yield much more compact expressions leading to a more efficient construction of the factorised squared matrix element, especially when considering spin-correlations.

Figure 1 shows the real and virtual contributions to a cross section up to $\mathrm{N}^{3} \mathrm{LO}$ and the primary singular limits which are either multiple soft, $S_{i_{1} \ldots i_{m}}$, or multiple collinear, $C_{i_{1} \ldots i_{m}}$. The factorisation amplitudes have been computed in all cases [9, 24, 26, 41-46] except for the triple-collinear and double-soft limits of the double-real-virtual. The triple collinear limit at one-loop has been considered at the squared amplitude level for $q \rightarrow q \bar{Q} Q$ [47] and for the mixed QCD+QED cases of $q \rightarrow q \gamma \gamma, q \rightarrow q g \gamma, g \rightarrow \bar{q} q \gamma, \gamma \rightarrow \bar{q} q \gamma$ and $\gamma \rightarrow \bar{q} q g[48,49]$.

In this article we compute the one-loop gluon initiated splitting functions in QCD, $g \rightarrow g g g$ and $g \rightarrow \bar{q} q g$. Splitting amplitudes valid in four dimensions are extracted from the known analytic amplitudes for $0 \rightarrow H g g g g \quad[50-54]$ and $0 \rightarrow H \bar{q} q g g \quad[50,55,56]$ in the large top-mass limit.

The structure of the article is as follows. We first introduce the notation for the amplitudes and the squared amplitudes together with their respective colour decompositions and collinear limits. In section 3 we describe a parametrisation of the multi-collinear limit using spinor-helicity variables which we will use to compute the splitting amplitudes. We then present the $g \rightarrow g g g$ and $g \rightarrow \bar{q} q g$ splitting amplitudes and describe the symmetries and super-symmetric decompositions used to obtain a compact representation. We then check the universality of the new splitting amplitudes by taking a numerical limit of the $g g \rightarrow g g g g$ and $g g \rightarrow \bar{q} q g g$ in NJET before reaching our conclusions. 


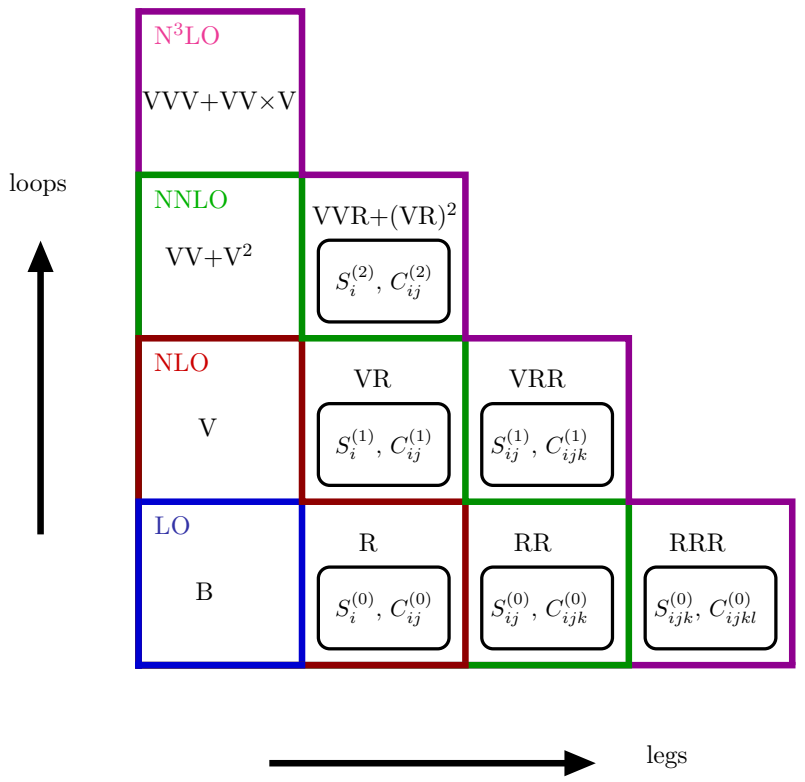

Figure 1. The contributions to perturbative cross sections up to $\mathrm{N}^{3} \mathrm{LO}$. This consists of virtual (V) corrections up to three loops and real radiation $(\mathrm{R})$ corrections with up to 3 additional unresolved legs. In the real radiation contributions the primary infrared limits of soft $(\mathrm{S})$ and collinear $(\mathrm{C})$ should be removed from the matrix elements and re-combined with the virtual corrections to obtain an infrared finite result.

\section{Notation}

A general QCD amplitude can be decomposed into a basis of $\mathrm{SU}\left(N_{c}\right)$ colour factors and ordered partial amplitudes which depend only on the momenta and helicities of the external legs. For an $n$-point $L$-loop amplitude this can be represented as,

$$
\mathcal{A}_{n}^{(L)}\left(\left\{a_{i}\right\},\left\{p_{i}^{\lambda_{i}}\right\}\right)=\sum_{c} C_{c}\left(\left\{a_{i}\right\}\right) A_{n ; c}^{(L)}\left(\left\{p_{i}^{\lambda_{i}}\right\}\right)
$$

where $a_{i}, \lambda_{i}$ and $p_{i}$ are colour indices (adjoint or fundamental), helicity and momenta of the $i^{\text {th }}$ leg. Unless explicitly indicated otherwise, we understand that the index $i$ runs from 1 to $n$, e.g.

$$
\left\{p_{i}^{\lambda_{i}}\right\} \equiv\left\{p_{i}^{\lambda_{i}}\right\}_{i=1}^{n}=\left\{p_{1}^{\lambda_{1}}, \ldots, p_{n}^{\lambda_{n}}\right\} .
$$

For cross-section computations we are required to square these amplitudes and sum over the colour indices. This sum can be represented as,

$$
\begin{aligned}
\mathcal{M}_{n}^{\left(L, L^{\prime}\right)}\left(\left\{p_{i}^{\lambda_{i}}\right\}\right) & =\sum_{a_{i}}\left(\mathcal{A}_{n}^{(L)}\left(\left\{a_{i}\right\},\left\{p_{i}^{\lambda_{i}}\right\}\right)\right)^{\dagger} \mathcal{A}_{n}^{\left(L^{\prime}\right)}\left(\left\{a_{i}\right\},\left\{p_{i}^{\lambda_{i}}\right\}\right) \\
& =\left(\vec{A}_{n}^{(L)}\left(\left\{p_{i}^{\lambda_{i}}\right\}\right)\right)^{\dagger} \cdot \mathcal{C}_{n}^{\left(L, L^{\prime}\right)} \cdot \vec{A}_{n}^{\left(L^{\prime}\right)}\left(\left\{p_{i}^{\lambda_{i}}\right\}\right),
\end{aligned}
$$

where the matrix $\mathcal{C}_{n}^{\left(L, L^{\prime}\right)}$ is a function of $N_{c}$ defined by

$$
\left(\mathcal{C}_{n}^{\left(L, L^{\prime}\right)}\right)_{c c^{\prime}}=\sum_{a_{i}}\left(C_{c}\left(\left\{a_{i}\right\}\right)\right)^{\dagger} C_{c^{\prime}}\left(\left\{a_{i}\right\}\right)
$$


while $\vec{A}^{(L)}$ is a vector of partial amplitudes $A_{n ; c}^{(L)}$

$$
\vec{A}^{(L)}=\left\{A_{n ; 1}^{(L)}, A_{n ; 2}^{(L)}, \ldots\right\} .
$$

Partial amplitudes may in turn be written in terms of primitive amplitudes $A_{p}^{[X]}$ which further decompose colour and flavour structure due to the internal loops,

$$
A_{n ; c}^{(L)}=\sum_{p, X} R_{c, p, X}\left(N_{c}, N_{f}\right) A_{n, p}^{[L, X]}
$$

where $X$ runs over the independent primitive topologies at $L$ loops and $p$ runs over permutations of the $n$ external legs. Eq. (2.3) can thus be equivalently written as

$$
\mathcal{M}_{n}^{\left(L, L^{\prime}\right)}\left(\left\{p_{i}^{\lambda_{i}}\right\}\right)=\left(\vec{A}_{n}^{[L]}\left(\left\{p_{i}^{\lambda_{i}}\right\}\right)\right)^{\dagger} \cdot \mathcal{C}_{n}^{\left[L, L^{\prime}\right]} \cdot \vec{A}_{n}^{\left[L^{\prime}\right]}\left(\left\{p_{i}^{\lambda_{i}}\right\}\right)
$$

where $\vec{A}_{n}^{[L]}$ is a vector of primitive amplitudes $A_{n, p}^{[L, X]}$ and the matrix $\mathcal{C}_{n}^{\left[L, L^{\prime}\right]}$ can be related to $\mathcal{C}_{n}^{\left(L, L^{\prime}\right)}$ defined in eq. (2.4) by the change of basis in eq. (2.6).

In the limit where $m$ of the external legs become simultaneously collinear, the amplitudes factorise into a product of lower multiplicity amplitudes and splitting amplitudes which contain all the infrared divergences:

$$
A_{n}^{(L)}\left(\left\{p_{i}^{\lambda_{i}}\right\}\right) \stackrel{1\|\ldots\| m}{\rightarrow} \sum_{k=0}^{L} \sum_{\lambda_{P}} \operatorname{Sp}_{m}^{(L-k)}\left(-P^{-\lambda_{P}} ;\left\{p_{i}^{\lambda_{i}}\right\}_{i=1}^{m}\right) A_{n-m+1}^{(k)}\left(P^{\lambda_{P}},\left\{p_{i}^{\lambda_{i}}\right\}_{i=m+1}^{n}\right)
$$

where $A_{n}^{(L)}$ and $\mathrm{Sp}_{n}^{(L)}$ can either be primitive or partial $n$-point amplitudes and splitting amplitudes respectively, while and $P \equiv p_{1}+\cdots+p_{m}$. A schematic representation of this factorisation is shown in figure 2. The sum of internal helicity states $\lambda_{P}$ leads to spin correlations in the factorized squared amplitude $\mathcal{M}^{\left(L, L^{\prime}\right)}$,

$$
\begin{aligned}
\mathcal{M}_{n}^{\left(L, L^{\prime}\right)}\left(\left\{p_{i}^{\lambda_{i}}\right\}\right) \stackrel{1\|\ldots\| m}{\rightarrow} & \sum_{k=0}^{L} \sum_{k^{\prime}=0}^{L^{\prime}} \sum_{\lambda_{P}, \lambda_{P}^{\prime}} \\
& \mathcal{P}_{m ;-\lambda_{P},-\lambda_{P}^{\prime}}^{\left(L-k, L^{\prime}-k^{\prime}\right)}\left(-P ;\left\{p_{i}^{\lambda_{i}}\right\}_{i=1}^{m}\right) \mathcal{M}_{n-m+1 ; \lambda_{P}, \lambda_{P}^{\prime}}^{\left(k, k^{\prime}\right)}\left(P,\left\{p_{i}^{\lambda_{i}}\right\}_{i=m+1}^{n}\right)
\end{aligned}
$$

where we can define

$$
\begin{aligned}
& \mathcal{M}_{n ; \lambda_{P}, \lambda_{P}^{\prime}}^{\left(L, L^{\prime}\right)}\left(P,\left\{p_{i}^{\lambda_{i}}\right\}\right)=\left(\vec{A}_{n}^{(L)}\left(P^{\lambda_{P}},\left\{p_{i}^{\lambda_{i}}\right\}\right)\right)^{\dagger} \cdot \mathcal{C}_{n}^{\left(L, L^{\prime}\right)} \cdot \vec{A}_{n}^{\left(L^{\prime}\right)}\left(P^{\lambda_{P^{\prime}}},\left\{p_{i}^{\lambda_{i}}\right\}\right) \\
& \mathcal{P}_{n ; \lambda_{P}, \lambda_{P}^{\prime}}^{\left(L, L^{\prime}\right)}\left(P ;\left\{p_{i}^{\lambda_{i}}\right\}\right)=\left(\overrightarrow{\mathrm{Sp}}_{n}^{(L)}\left(P^{\lambda_{P}} ;\left\{p_{i}^{\lambda_{i}}\right\}\right)\right)^{\dagger} \cdot \mathcal{C}_{\mathrm{Sp}, n}^{\left(L, L^{\prime}\right)} \cdot \overrightarrow{\mathrm{Sp}}_{n}^{\left(L^{\prime}\right)}\left(P^{\lambda_{P^{\prime}}} ;\left\{p_{i}^{\lambda_{i}}\right\}\right)
\end{aligned}
$$

in terms of partial amplitudes or equivalently

$$
\begin{aligned}
& \mathcal{M}_{n ; \lambda_{P}, \lambda_{P}^{\prime}}^{\left(L, L^{\prime}\right)}\left(P,\left\{p_{i}^{\lambda_{i}}\right\}\right)=\left(\vec{A}_{n}^{[L]}\left(P^{\lambda_{P}},\left\{p_{i}^{\lambda_{i}}\right\}\right)\right)^{\dagger} \cdot \mathcal{C}_{n}^{\left[L, L^{\prime}\right]} \cdot \vec{A}_{n}^{\left[L^{\prime}\right]}\left(P^{\lambda_{P^{\prime}}},\left\{p_{i}^{\lambda_{i}}\right\}\right) \\
& \mathcal{P}_{n ; \lambda_{P}, \lambda_{P}^{\prime}}^{\left(L, L^{\prime}\right)}\left(P ;\left\{p_{i}^{\lambda_{i}}\right\}\right)=\left({\overrightarrow{\mathrm{Sp}_{n}}}^{[L]}\left(P^{\lambda_{P}} ;\left\{p_{i}^{\lambda_{i}}\right\}\right)\right)^{\dagger} \cdot \mathcal{C}_{\mathrm{Sp}, n}^{\left[L, L^{\prime}\right]} \cdot \overrightarrow{\mathrm{Sp}}_{n}^{\left[L^{\prime}\right]}\left(P^{\lambda_{P^{\prime}}} ;\left\{p_{i}^{\lambda_{i}}\right\}\right)
\end{aligned}
$$




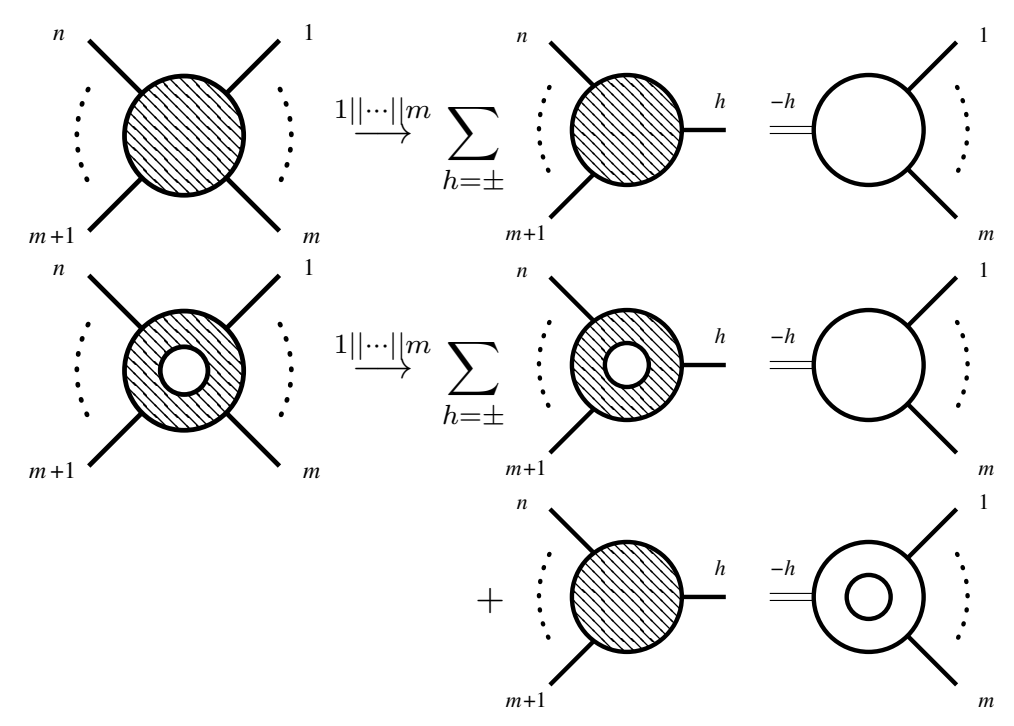

Figure 2. Factorisation of tree and one-loop amplitudes in the multi-collinear limit.

in terms of primitive amplitudes. In the colour matrix $\mathcal{C}_{\mathrm{Sp}, n}^{\left[L, L^{\prime}\right]}$ we absorbed a prefactor which takes into account colour conservation along the factorized parton, such that

$$
\mathcal{C}_{\mathrm{Sp}, n}^{\left[L, L^{\prime}\right]}=\left\{\begin{array}{ll}
\frac{1}{N_{c}^{2}-1} \mathcal{C}_{n}^{\left[L, L^{\prime}\right]} & \text { for gluon-initiated Sp } \\
\frac{1}{N_{c}} \mathcal{C}_{n}^{\left[L, L^{\prime}\right]} & \text { for quark-initiated Sp }
\end{array},\right.
$$

and similar for $\mathcal{C}_{\mathrm{Sp}, n}^{\left(L, L^{\prime}\right)}$.

For brevity, the results presented in this paper will often omit the subscript indicating the number of partons involved in an amplitude, since this can be deduced by its arguments, i.e.

$$
\begin{aligned}
\operatorname{Sp}\left(-P^{\lambda_{P}} ; p_{1}^{\lambda_{1}}, \ldots, p_{n}^{\lambda_{n}}\right) & \equiv \operatorname{Sp}_{n}\left(-P^{\lambda_{P}} ;\left\{p_{i}^{\lambda_{i}}\right\}\right) \\
A\left(p_{1}^{\lambda_{1}}, \ldots, p_{n}^{\lambda_{n}}\right) & \equiv A_{n}\left(\left\{p_{i}^{\lambda_{i}}\right\}\right) .
\end{aligned}
$$

\section{A spinor parametrisation of the multi-collinear limit}

We define the multiple collinear limit using a parametrisation of the full kinematics in term of a parameter $\delta$, such that the collinear limit in eq. (2.8) is identified as the leading term as $\delta \rightarrow 0$, i.e.

$$
\begin{aligned}
& \lim _{1\|\cdots\| m} A_{n}^{(L)}\left(\left\{p_{i}^{\lambda_{i}}\right\}\right)=\lim _{\delta \rightarrow 0} A_{n}^{(L)}\left(\left\{p_{i}^{\lambda_{i}}(\delta)\right\}\right) \\
& =\sum_{k=0}^{L} \sum_{\lambda_{P}} \operatorname{Sp}_{m}^{(L-k)}\left(-P^{-\lambda_{P}} ;\left\{p_{i}^{\lambda_{i}}\right\}_{i=1}^{m}\right) A_{n-m+1}^{(k)}\left(P^{\lambda_{P}},\left\{p_{i}^{\lambda_{i}}\right\}_{i=m+1}^{n}\right)+\mathcal{O}\left(\frac{1}{\delta^{m-2}}\right)
\end{aligned}
$$


The parametrisation is defined by,

$$
\begin{array}{ll}
p_{i}^{\mu}(\delta)=z_{i} \tilde{P}^{\mu}+\delta k_{T, i}^{\mu}-\delta^{2} \frac{k_{T, i}^{2}}{2(P \cdot \eta) z_{i}} \eta^{\mu} & i=1, \ldots, m \\
p_{i}^{\mu}(\delta)=K_{i}^{\mu}\left(\delta,\{p\}_{m+1, n}, \eta\right) & i=m+1, \ldots, n
\end{array}
$$

where $z_{i}=\left(p_{i} \cdot \eta\right) /(P \cdot \eta)$ are the momentum fractions of the unresolved partons, $\eta$ is an arbitrary light-like momentum and $\tilde{P}$ is the massless projection of $P=\sum_{i=1}^{m} p_{i}$,

$$
\tilde{P}^{\mu}=P^{\mu}-\frac{P^{2}}{2 P \cdot \eta} \eta^{\mu}
$$

The vectors $k_{T, i}^{\mu}$ are orthogonal to $P, \tilde{P}$ and $\eta$

$$
k_{T, i} \cdot \tilde{P}=k_{T, i} \cdot P=k_{T, i} \cdot \eta=0
$$

Momentum conservation implies that:

$$
\begin{gathered}
\sum_{i=1}^{m} z_{i}=1 \\
\sum_{i=1}^{m} k_{T, i}^{\mu}=0^{\mu} \\
-\delta^{2} \sum_{i=1}^{m} \frac{k_{T, i}^{2}}{z_{i}}=P^{2} .
\end{gathered}
$$

The function $K_{i}^{\mu}$ is a generic map that keeps the factorized momenta $m+1, \ldots, n$ on-shell as well as absorbing the recoil $P^{2} /(2 P \cdot \eta) \eta^{\mu}$, and it satisfies $K_{i}^{\mu} \rightarrow p_{i}^{\mu}$ as $\delta \rightarrow 0$. The exact form is not important for our purpose of explicitly taking the limit and various mappings have been considered in the literature (for example in the Catani-Seymour subtraction [1] or Kosower's antenna [57]). When implementing the collinear phase-space numerically we employed the Catani-Seymour map as described in appendix A.

Since we are working at the amplitude level, we would like to have a parametrisation of the limit valid for the spinors of $p_{i}$ as well. This can be achieved using an appropriate choice of the transverse vectors $k_{T, i}$,

$$
2 \delta k_{T, i}^{\mu}=\left\langle z_{i}\right\rangle\left[\omega_{i}\right]\left\langle\tilde{P}\left|\gamma^{\mu}\right| \eta\right]+\left[z_{i}\right]\left\langle\omega_{i}\right\rangle\left\langle\eta\left|\gamma^{\mu}\right| \tilde{P}\right] .
$$

In the above we use the notation

$$
\left\langle z_{i}\right\rangle=\frac{\langle i \eta\rangle}{\langle\tilde{P} \eta\rangle}, \quad\left[z_{i}\right]=\frac{[i \eta]}{[\tilde{P} \eta]}, \quad\left\langle\omega_{i}\right\rangle=\frac{\langle i \tilde{P}\rangle}{\langle\eta \tilde{P}\rangle}, \quad\left[\omega_{i}\right]=\frac{[i \tilde{P}]}{[\eta \tilde{P}]},
$$

where the spinor variables $\left\langle z_{i}\right\rangle$ and $\left[z_{i}\right]$ differ by a phase from the usual parametrisation which uses $\sqrt{z_{i}}$. It is worth to notice that both $\left\langle\omega_{i}\right\rangle$ and $\left[\omega_{i}\right]$ are $\mathcal{O}(\delta)$ in the collinear limit. The spinors parametrisation then reads,

$$
\left.\left.\left.|i\rangle=\left\langle z_{i}\right\rangle|\tilde{P}\rangle+\left\langle\omega_{i}\right\rangle|\eta\rangle \quad \quad \mid i\right]=\left[z_{i}\right] \mid \tilde{P}\right]+\left[\omega_{i}\right] \mid \eta\right]
$$


We find that this is a convenient way to take the limit at the amplitude level since the spinor variables $\left\langle z_{i}\right\rangle$ obey Schouten identities:

$$
\sum_{i j k \text { cyclic }}\left\langle z_{i}\right\rangle\langle j k\rangle=0
$$

as well as momentum conservation,

$$
\sum_{i} p_{i}^{\mu}-\tilde{P}^{\mu}-\frac{P^{2}}{2 P \cdot \eta} \eta^{\mu}=0^{\mu}
$$

For the triple collinear splitting amplitudes this means we have the kinematics of a fivepoint function event though the colour space is that of a four-point function.

\subsection{Example: the tree-level MHV multi-collinear splitting amplitude}

The result for the multi-collinear limit of the maximal-helicity-violating (MHV) amplitude has been known for a long time. More recently the general helicity cases were also examined through use of the MHV rules [45, 46]. This case is incredibly straightforward and serves as a useful example of the general treatment introduced in the previous section.

We start with the Parke-Taylor MHV amplitude with particles 1 and $r>m$ having negative helicities and all others positive helicity,

$$
A_{n}^{(0)}\left(1^{-}, 2^{+}, 3^{+}, \ldots, r^{-}, \ldots, n^{+}\right)=\frac{\langle 1 r\rangle^{4}}{\prod_{i=1}^{n}\langle i i+1\rangle},
$$

where the product in the denominator is considered modulo $n$. The limit is simply taken by applying eq. (3.11)

$$
\begin{aligned}
& A_{n}^{(0)}\left(1^{-}, 2^{+}, 3^{+}, \ldots, r^{-}, \ldots, n^{+}\right)= \\
& \frac{\left(\left\langle z_{1}\right\rangle\left\langle\tilde{P}_{1, m} r\right\rangle+\left\langle\omega_{1}\right\rangle\langle\eta r\rangle\right)^{4}}{\prod_{j=1}^{m-1}\langle j j+1\rangle\left(\left\langle z_{1}\right\rangle\left\langle n \tilde{P}_{1, m}\right\rangle+\left\langle\omega_{1}\right\rangle\langle n \eta\rangle\right)\left(\left\langle z_{m}\right\rangle\left\langle\tilde{P}_{1, m} m+1\right\rangle+\left\langle\omega_{m}\right\rangle\langle\eta m+1\rangle\right) \prod_{i=r}^{n-1}\langle i i+1\rangle} \\
& \stackrel{\langle\rightarrow 0}{\stackrel{\left\langle z_{1}\right\rangle^{3}}{\left\langle z_{m}\right\rangle \prod_{j=1}^{m-1}\langle j j+1\rangle} \frac{\left\langle\tilde{P}_{1, m} r\right\rangle^{4}}{\left\langle n \tilde{P}_{1, m}\right\rangle\left\langle\tilde{P}_{1, m} m+1\right\rangle \prod_{i=m+1}^{n-1}\langle i i+1\rangle}+\mathcal{O}\left(\delta^{3-m}\right)} \\
& =\operatorname{Sp}^{(0)}\left(-\tilde{P}_{1, m}^{+} ; 1^{-}, 2^{+}, \ldots, m^{+}\right) A_{n-m+1}^{(0)}\left(\tilde{P}_{1, m}^{-},(m+1)^{+}, \ldots, r^{-}, \ldots, n^{+}\right)+\mathcal{O}\left(\delta^{3-m}\right)
\end{aligned}
$$

where we have used eq. (3.9) to perform the power counting. For $i, j \in[1, m]$ this can be seen explicitly,

$$
\begin{aligned}
\left\langle\omega_{i}\right\rangle & =-\delta \frac{\left\langle\tilde{P}_{1, m}\left|k_{T}\right| \eta\right]}{2\left(P_{1, m} \cdot \eta\right)\left[z_{j}\right]}=\mathcal{O}(\delta) \\
\Rightarrow\langle i j\rangle & =\left(\left\langle z_{i}\right\rangle\left\langle\omega_{j}\right\rangle-\left\langle z_{j}\right\rangle\left\langle\omega_{i}\right\rangle\right)\left\langle\tilde{P}_{1, m} \eta\right\rangle=\mathcal{O}(\delta) .
\end{aligned}
$$

One can clearly arrive at this final result without being so explicit about the parametrisation, yet it is convenient to have one in a generic implementation. 


\subsection{One-loop basis functions for $p p \rightarrow H+2 j$ in the triple collinear limit}

The analytic $H+4$ parton amplitudes have been computed using unitarity cuts and expressed in terms of the universal infrared poles plus finite logarithmic and di-logarithmic functions as well as rational terms. Taking the triple collinear limit of the infrared poles, rational terms and logarithms as above presents no difficulties. Dealing with the dilogarithmic parts requires some minor effort to ensure the arguments are in the appropriate region so the limit will converge. Polylogarithmic identities are well known and understood in huge detail (see ref. [58] for a recent review) - way beyond the simple structures appearing here. Nevertheless we collect some potentially useful identities here to aid the reader,

$$
\begin{array}{cc}
\operatorname{Li}_{2}(1-x)+\operatorname{Li}_{2}(x)+\log (x) \log (1-x)-\frac{\pi^{2}}{6}=0 & x \in[0,1] \\
\operatorname{Li}_{2}(x)+\operatorname{Li}_{2}\left(\frac{1}{x}\right)+\frac{1}{2} \log (-x)^{2}+\frac{\pi^{2}}{6}=0 & x<0 \\
\operatorname{Li}_{2}\left(\frac{x y}{(1-x)(1-y)}\right)-\operatorname{Li}_{2}\left(-\frac{x}{1-x}\right)-\operatorname{Li}_{2}\left(-\frac{y}{1-y}\right)+ & \\
-\mathrm{Li}_{2}\left(\frac{x}{1-y}\right)-\mathrm{Li}_{2}\left(\frac{y}{1-x}\right)-\log ^{2}\left(\frac{1-x}{1-y}\right) & =0 \quad x, y \in[0,1]
\end{array}
$$

One function requiring a bit more thought is the three mass triangle which has square roots appearing in the arguments of the di-logarithms [59-63]:

$$
\begin{aligned}
\mathrm{I}_{3}^{3 m}\left(s_{i j}, s_{k l}, m_{H}^{2}\right) \stackrel{i\|j\| k}{\longrightarrow} & \frac{1}{\left(1-z_{k}\right) m_{H}^{2}}\left(\operatorname{Li}_{2}\left(1-z_{k}\right)-\operatorname{Li}_{2}\left(1-\frac{1}{z_{k}}\right)\right. \\
& \left.-\frac{1}{2} \log ^{2}\left(z_{k}\right)-\log \left(\frac{m_{H}^{2}}{s_{i j}}\right) \log \left(z_{k}\right)\right)
\end{aligned}
$$

One other minor issue with the results available in the literature is that the NMHV expressions have been presented using Forde's method for triple cuts [64]. This method gives the coefficients as the sum over solutions to the on-shell equations. To aid our computation we performed this sum explicitly to write the coefficients in terms of the usual spinor products of the external momenta.

\section{$4 \quad g \rightarrow g g g$ splitting amplitudes}

\subsection{Colour structure and primitive amplitude decomposition}

In the section we will suppress all helicity superscripts and the function arguments are taken to represent both momenta and helicity. The tree-level colour decomposition can be 
written as,

$$
\begin{aligned}
\mathcal{S p}^{(0)} & \left(\left\{a_{P}, a_{1}, a_{2}, a_{3}\right\},-P ; 1,2,3\right) \\
& =\sum_{\sigma \in S_{3}} \operatorname{tr}\left(a_{P}, a_{\sigma(1)}, a_{\sigma(2)}, a_{\sigma(3)}\right) \operatorname{Sp}^{(0)}(-P ; \sigma(1), \sigma(2), \sigma(3)) \\
& =\sum_{\sigma \in S_{2}} \tilde{f}^{a_{1} a_{\sigma(2)} b} \tilde{f}^{b a_{\sigma(3)} a_{P}} \operatorname{Sp}^{(0)}(-P ; 1, \sigma(2), \sigma(3))
\end{aligned}
$$

where $\operatorname{tr}\left(a_{1}, \ldots, a_{n}\right)=T_{j i_{1}}^{a_{1}} T_{i_{1} i_{2}}^{a_{2}} \ldots T_{i_{n-1} j}^{a_{n}}$ in terms of the fundamental generators of $\mathrm{SU}\left(N_{c}\right)$ and $\tilde{f}^{a b c}=i \sqrt{2} f^{a b c}$ in terms of the adjoint structure constants. The relation between the two representations can be shown to hold using the Kleiss-Kuijf relations [65] for the splitting amplitudes,

$$
\begin{aligned}
& \mathrm{Sp}^{(0)}(-P ; 3,2,1)=\mathrm{Sp}^{(0)}(-P ; 1,2,3) \\
& \mathrm{Sp}^{(0)}(-P ; 2,1,3)=-\mathrm{Sp}^{(0)}(-P ; 1,2,3)-\mathrm{Sp}^{(0)}(-P ; 1,3,2)
\end{aligned}
$$

The one-loop colour decomposition is, ${ }^{1}$

$$
\begin{aligned}
\mathcal{S p}^{(1)} & \left(\left\{a_{P}, a_{1}, a_{2}, a_{3}\right\},-P ; 1,2,3\right) \\
= & \sum_{\sigma \in S_{3}} \operatorname{tr}\left(a_{P}, a_{\sigma(1)}, a_{\sigma(2)}, a_{\sigma(3)}\right) \mathrm{Sp}_{1}^{(1)}(-P ; \sigma(1), \sigma(2), \sigma(3)) \\
& +\sum_{\sigma \in S_{3} / Z_{2}} \operatorname{tr}\left(a_{P}, a_{\sigma(1)} \operatorname{tr}\left(a_{\sigma(2)}, a_{\sigma(3)}\right) \operatorname{Sp}_{3}^{(1)}(-P ; \sigma(1), \sigma(2), \sigma(3))\right.
\end{aligned}
$$

where the partial amplitudes are composed of primitive amplitudes as follows:

$$
\begin{aligned}
& \mathrm{Sp}_{1}^{(1)}(-P ; 1,2,3) \\
& =N_{c} \mathrm{Sp}^{[g]}(-P ; 1,2,3)-N_{f} \operatorname{Sp}^{[f]}(-P ; 1,2,3) \\
& \mathrm{Sp}_{3}^{(1)}(-P ; 1,2,3) \\
& =2\left(\mathrm{Sp}^{[g]}(-P ; 1,2,3)+\mathrm{Sp}^{[g]}(-P ; 1,3,2)+\mathrm{Sp}^{[g]}(-P ; 3,1,2)\right) .
\end{aligned}
$$

The primitive amplitudes for the gluon and fermion loops obey line-reversal symmetry,

$$
\mathrm{Sp}^{[X]}(-P ; 1,2,3)=\mathrm{Sp}^{[X]}(-P ; 3,2,1)
$$

and so in all we have three independent gluon loop primitive amplitudes, three fermion loop primitive amplitudes and two tree-level primitive amplitudes. The colour summed

\footnotetext{
${ }^{1}$ We write the one-loop decomposition in the standard trace basis rather than the slightly more compact 'F-basis' representation of Del Duca-Maltoni-Dixon [66]. Since we express the colour summed squared matrix element in terms of the minimal basis of primitive amplitudes the final expressions are equivalent to the DDM forms.
} 
Born and virtual corrections can then be written according to (2.13) using:

$$
\begin{aligned}
& \overrightarrow{\mathrm{Sp}}^{[0]}=\left(\begin{array}{l}
\mathrm{Sp}^{[0]}(-P ; 1,2,3) \\
\mathrm{Sp}^{[0]}(-P ; 1,3,2)
\end{array}\right) \\
& \mathcal{C}_{\mathrm{Sp}}^{[0,0]}=N_{c}^{2}\left(\begin{array}{ll}
4 & 2 \\
2 & 4
\end{array}\right) \\
& \overrightarrow{\mathrm{Sp}^{[1]}}=\left(\begin{array}{c}
N_{c} \mathrm{Sp}^{[g]}(-P ; 1,2,3) \\
N_{c} \mathrm{Sp}^{[g]}(-P ; 2,1,3) \\
N_{c} \mathrm{Sp}^{[g]}(-P ; 2,3,1) \\
N_{f} \mathrm{Sp}^{[f]}(-P ; 1,2,3) \\
N_{f} \mathrm{Sp}^{[f]}(-P ; 2,1,3) \\
N_{f} \mathrm{Sp}^{[f]}(-P ; 2,3,1)
\end{array}\right) \\
& \mathcal{C}_{\mathrm{Sp}}^{[0,1]}=2 N_{c}^{2}\left(\begin{array}{cccccc}
2 & -2 & 0 & -2 & 2 & 0 \\
0 & -2 & 2 & 0 & 2 & -2
\end{array}\right)
\end{aligned}
$$

We also choose to present the results using the super-symmetric decomposition:

$$
\begin{aligned}
& \operatorname{Sp}^{[g]}(-P ; 1,2,3) \\
& =\operatorname{Sp}^{[\mathcal{N}=4]}(-P ; 1,2,3)+4 \operatorname{Sp}^{[\mathcal{N}=1]}(-P ; 1,2,3)+\left(1-\epsilon \delta_{R}\right) \operatorname{Sp}^{[\mathcal{N}=0]}(-P ; 1,2,3) \\
& \operatorname{Sp}^{[f]}(-P ; 1,2,3) \\
& =\operatorname{Sp}^{[\mathcal{N}=1]}(-P ; 1,2,3)+\operatorname{Sp}^{[\mathcal{N}=0]}(-P ; 1,2,3)
\end{aligned}
$$

since this yields particularly compact expressions. We also include the scheme dependence for both the $\mathrm{FDH}\left(\delta_{R}=0\right)$ and $\operatorname{CDR}\left(\delta_{R}=1\right)$ schemes.

\subsection{Results}

We define the following phase-free quantities,

$$
\alpha_{i j} \equiv \alpha_{i j k}=\frac{\langle i j\rangle\left\langle z_{k}\right\rangle}{\langle j k\rangle\left\langle z_{i}\right\rangle}, \quad \beta_{i j} \equiv \beta_{i j k}=\frac{[i j]\left[\omega_{k}\right]}{[j k]\left[\omega_{i}\right]}, \quad \gamma_{i j}=\frac{\left\langle z_{i}\right\rangle[i j]}{[j \tilde{P}]} .
$$

Since there can be no repeated index in either $\alpha_{i j k}$ and $\beta_{i j k}$ each can be uniquely specified by the two first labels.

The integral functions are defined using the following basis,

$$
\begin{aligned}
F^{\mathrm{MHV}}= & \frac{1}{2}\left(\log ^{2}\left(z_{1}\right)+\log ^{2}\left(z_{3}\right)+\frac{\pi^{2}}{3}\right)-\log \left(\frac{s_{12}}{s_{123}}\right) \log \left(\frac{s_{23}}{s_{123}}\right) \\
& +\log \left(\frac{1-z_{3}}{z_{1}}\right) \log \left(\frac{s_{12}}{s_{123}}\right)+\log \left(\frac{1-z_{1}}{z_{3}}\right) \log \left(\frac{s_{23}}{s_{123}}\right) \\
& +\operatorname{Li}_{2}\left(-\frac{z_{2}}{z_{1}}\right)+\operatorname{Li}_{2}\left(-\frac{z_{2}}{z_{3}}\right)+\operatorname{Li}_{2}\left(-\frac{z_{3}}{1-z_{3}}\right)+\operatorname{Li}_{2}\left(-\frac{z_{1}}{1-z_{1}}\right) \\
& -\operatorname{Li}_{2}\left(1-\frac{s_{12}}{\left(1-z_{3}\right) s_{123}}\right)-\operatorname{Li}_{2}\left(1-\frac{s_{23}}{\left(1-z_{1}\right) s_{123}}\right)
\end{aligned}
$$




$$
\begin{aligned}
F_{1}^{\mathrm{NMHV}}= & -\log \left(1-z_{3}\right)\left(\log \left(\frac{z_{1} z_{3}}{1-z_{3}}\right)+\log \left(\frac{s_{12}}{s_{23}}\right)\right)+\log \left(z_{1} z_{3}\right) \log \left(\frac{s_{12}}{s_{123}}\right) \\
& -\frac{1}{2}\left(\log \left(z_{3}\right) \log \left(\frac{s_{12}}{s_{123}}\right)+\log \left(z_{1}\right) \log \left(\frac{s_{23}}{s_{123}}\right)-\frac{\pi^{2}}{3}\right) \\
F_{2}^{\mathrm{NMHV}=} & \left.F_{1}^{\mathrm{NMHV}}\right|_{1 \leftrightarrow 3} \\
F_{3}^{\mathrm{NMHV}}= & \frac{1}{2}\left(\log \left(z_{3}\right) \log \left(\frac{s_{12}}{s_{123}}\right)+\log \left(z_{1}\right) \log \left(\frac{s_{23}}{s_{123}}\right)-\frac{\pi^{2}}{3}\right) \\
& -\log \left(\frac{s_{12}}{s_{123}}\right) \log \left(\frac{s_{23}}{s_{123}}\right) \\
F_{\mathrm{box}}^{1 \mathrm{~m}}= & -\frac{\pi^{2}}{3}-\log { }^{2}\left(\frac{s_{12}}{s_{23}}\right)-2\left(\operatorname{Li}_{2}\left(1-\frac{s_{123}}{s_{12}}\right)+\mathrm{Li}_{2}\left(1-\frac{s_{123}}{s_{23}}\right)\right) \\
\hat{L}_{0}\left(s_{1}, s_{2}\right)= & \log \left(\frac{s_{1}}{s_{2}}\right) \\
\hat{L}_{1}\left(s_{1}, s_{2}\right)= & \frac{1}{s_{1}-s_{2}} \log \left(\frac{s_{1}}{s_{2}}\right) \\
\hat{L}_{2}\left(s_{1}, s_{2}\right)= & \frac{1}{\left(s_{1}-s_{2}\right)^{2}} \log \left(\frac{s_{1}}{s_{2}}\right)-\frac{1}{2} \frac{1}{s_{1}-s_{2}}\left(\frac{1}{s_{1}}+\frac{1}{s_{2}}\right) \\
\hat{L}_{3}\left(s_{1}, s_{2}\right)= & \frac{1}{\left(s_{1}-s_{2}\right)^{3}} \log \left(\frac{s_{1}}{s_{2}}\right)-\frac{1}{2} \frac{1}{\left(s_{1}-s_{2}\right)^{2}}\left(\frac{1}{s_{1}}+\frac{1}{s_{2}}\right)
\end{aligned}
$$

We express the infrared poles and associated logarithms as described by Catani's formula [47],

$$
V_{g}=-\frac{1}{\epsilon^{2}}\left(\left(\frac{\mu_{R}}{-s_{12}}\right)^{\epsilon}+\left(\frac{\mu_{R}}{-s_{23}}\right)^{\epsilon}+\left(\frac{\mu_{R}}{-s_{123}}\right)^{\epsilon}\left(z_{1}^{-\epsilon}+z_{3}^{-\epsilon}-2\right)\right)
$$

All results in this section are presented unrenormalized.

The tree-level splitting amplitudes are,

$$
\begin{aligned}
\mathrm{Sp}^{(0)}\left(-P^{+} ; 1^{+}, 2^{+}, 3^{+}\right) & =0 \\
\mathrm{Sp}^{(0)}\left(-P^{+} ; 1^{-}, 2^{-}, 3^{-}\right) & =\frac{1}{\left[z_{1}\right]\left[z_{3}\right][12][23]} \\
\mathrm{Sp}^{(0)}\left(-P^{+} ; 1^{+}, 2^{+}, 3^{-}\right) & =\frac{\left\langle z_{3}\right\rangle^{3}}{\left\langle z_{1}\right\rangle\langle 12\rangle\langle 23\rangle} \\
\mathrm{Sp}^{(0)}\left(-P^{+} ; 1^{+}, 2^{-}, 3^{+}\right) & =\frac{\left\langle z_{2}\right\rangle^{4}}{\left\langle z_{1}\right\rangle\left\langle z_{3}\right\rangle\langle 12\rangle\langle 23\rangle} \\
\mathrm{Sp}^{(0)}\left(-P^{+} ; 1^{+}, 2^{-}, 3^{-}\right) & =-\frac{[1 P]^{2}}{[23]^{2}}\left(\frac{\beta_{32}}{s_{123}}+\frac{\gamma_{23}^{3} \alpha_{12} \beta_{12}^{2} s_{23}}{\left(1-z_{3}\right) z_{3} s_{12}^{2}}\right) \\
\mathrm{Sp}^{(0)}\left(-P^{+} ; 1^{-}, 2^{+}, 3^{-}\right) & =-\frac{[2 P]^{2}}{[13]^{2} s_{1 P} s_{3 P}}( \\
\frac{\beta_{21} \beta_{23} s_{13}^{4}}{s_{12} s_{123} s_{23}} & \left.+\frac{\gamma_{32}^{2} z_{2} z_{3} s_{1 P}\left(\alpha_{13} \alpha_{31}\right)^{\dagger}}{\gamma_{23}\left(1-z_{3}\right)}+\frac{\gamma_{12}^{2} z_{1} z_{2} s_{3 P}\left(\alpha_{13} \alpha_{31}\right)^{\dagger}}{\gamma_{21}\left(1-z_{1}\right)}\right)
\end{aligned}
$$


All other helicity configurations are given via parity or the line-reversal symmetry of eq. (4.8). The one-loop splitting primitive amplitudes are,

$$
\begin{aligned}
& \mathrm{Sp}^{[\mathcal{N}=4]}\left(-P^{+} ; 1^{+}, 2^{+}, 3^{+}\right)=0 \\
& \mathrm{Sp}^{[\mathcal{N}=4]}\left(-P^{+} ; 1^{-}, 2^{-}, 3^{-}\right)=\mathrm{Sp}^{(0)}\left(-P^{+} ; 1^{-}, 2^{-}, 3^{-}\right)\left(V_{g}+F^{\mathrm{MHV}}\right) \\
& \mathrm{Sp}^{[\mathcal{N}=4]}\left(-P^{+} ; 1^{+}, 2^{+}, 3^{-}\right)=\mathrm{Sp}^{(0)}\left(-P^{+} ; 1^{+}, 2^{+}, 3^{-}\right)\left(V_{g}+F^{\mathrm{MHV}}\right) \\
& \mathrm{Sp}^{[\mathcal{N}=4]}\left(-P^{+} ; 1^{+}, 2^{-}, 3^{+}\right)=\mathrm{Sp}^{(0)}\left(-P^{+} ; 1^{+}, 2^{-}, 3^{+}\right)\left(V_{g}+F^{\mathrm{MHV}}\right) \\
& \mathrm{Sp}^{[\mathcal{N}=4]}\left(-P^{+} ; 1^{+}, 2^{-}, 3^{-}\right)=\mathrm{Sp}^{(0)}\left(-P^{+} ; 1^{+}, 2^{-}, 3^{-}\right)\left(V_{g}\right)-\frac{[1 P]^{2}}{[23]^{2}}( \\
& +\left(\frac{\gamma_{23} \alpha_{12} \beta_{12} s_{23}}{z_{3} s_{12}^{2}}\left(\frac{s_{12}\left(z_{1}-1\right)^{3}}{z_{2} s_{1 P}}+\frac{2 \gamma_{23}^{2} \beta_{12}}{z_{3}-1}\right)+\frac{1}{s_{123}}\left(\frac{\beta_{12} s_{23}^{3}}{s_{12} s_{1 P} s_{3 P}}-\beta_{32}\right)\right) F_{1}^{\mathrm{NMHV}} \\
& -\frac{\gamma_{23} \alpha_{12} \beta_{12} s_{23}\left(z_{1}-1\right)^{3}}{z_{2} z_{3} s_{12} s_{1 P}} F_{2}^{\mathrm{NMHV}} \\
& \left.+\frac{1}{s_{123}}\left(\frac{\beta_{12} s_{23}^{3}}{s_{12} s_{1 P} s_{3 P}}+\beta_{32}\right) F_{3}^{\mathrm{NMHV}}\right) \\
& \mathrm{Sp}^{[\mathcal{N}=4]}\left(-P^{+} ; 1^{-}, 2^{+}, 3^{-}\right)=\mathrm{Sp}^{(0)}\left(-P^{+} ; 1^{-}, 2^{+}, 3^{-}\right)\left(V_{g}\right)-\frac{[2 P]^{2}}{[13]^{2}}( \\
& +\left(\frac{\gamma_{32} \beta_{31} s_{13} \gamma_{12}^{2}}{\gamma_{23} z_{3} s_{12} s_{3 P}}+\frac{z_{2} z_{3}\left(\alpha_{13} \alpha_{31}\right)^{\dagger} \gamma_{32}^{2}}{\gamma_{23} s_{3 P}\left(z_{3}-1\right)}+\frac{\gamma_{12}^{2} z_{2} \beta_{31}^{2} \gamma_{32}^{2}}{\gamma_{23}^{2} z_{3} s_{3 P}\left(z_{3}-1\right)}\right) F_{1}^{\mathrm{NMHV}} \\
& +\left(\frac{z_{1}\left(\alpha_{13} \alpha_{31}\right)^{\dagger} \gamma_{12}^{2}}{s_{1 P}}+\frac{\gamma_{12} \beta_{13} \beta_{31} \gamma_{32}^{3}}{\gamma_{23} z_{1} s_{23}\left(z_{1}-1\right)}+\frac{\gamma_{12} z_{1}^{2}\left(\alpha_{13} \alpha_{31}\right)^{\dagger} \gamma_{32}}{s_{1 P}\left(z_{1}-1\right)}\right) F_{2}^{\mathrm{NMHV}} \\
& \left.+\frac{1}{s_{123}}\left(\frac{\beta_{21} \beta_{23} s_{13}^{4}}{s_{12} s_{23} s_{1 P} s_{3 P}}+\beta_{13} \beta_{31}\right) F_{3}^{\mathrm{NMHV}}\right) \\
& \mathrm{Sp}^{[\mathcal{N}=1]}\left(-P^{+} ; 1^{+}, 2^{+}, 3^{+}\right)=0 \\
& \mathrm{Sp}^{[\mathcal{N}=1]}\left(-P^{+} ; 1^{-}, 2^{-}, 3^{-}\right)=0 \\
& \mathrm{Sp}^{[\mathcal{N}=1]}\left(-P^{+} ; 1^{+}, 2^{+}, 3^{-}\right)=\mathrm{Sp}^{(0)}\left(-P^{+} ; 1^{+}, 2^{+}, 3^{-}\right) \alpha_{32} s_{12} \hat{L}_{1}\left(s_{23}, s_{123}\right) \\
& \mathrm{Sp}^{[\mathcal{N}=1]}\left(-P^{+} ; 1^{+}, 2^{-}, 3^{+}\right)=\mathrm{Sp}^{(0)}\left(-P^{+} ; 1^{+}, 2^{-}, 3^{+}\right) \frac{\alpha_{23}}{\alpha_{31}}( \\
& \left.\frac{F_{\text {box }}^{1 m}}{2}-\left(\hat{L}_{1}\left(s_{12}, s_{123}\right)+\hat{L}_{1}\left(s_{23}, s_{123}\right)\right) s_{13}\right) \\
& \mathrm{Sp}^{[\mathcal{N}=1]}\left(-P^{+} ; 1^{+}, 2^{-}, 3^{-}\right)=-\frac{[1 P]^{2} s_{23}}{[23]^{2} s_{123}} \hat{L}_{1}\left(s_{12}, s_{123}\right) \\
& \mathrm{Sp}^{[\mathcal{N}=1]}\left(-P^{+} ; 1^{-}, 2^{+}, 3^{-}\right)=-\frac{[2 P]^{2}}{[13]^{2} s_{123}}( \\
& \left.\frac{F_{\text {box }}^{1 \mathrm{~m}}}{2}-\left(\hat{L}_{1}\left(s_{12}, s_{123}\right)+\hat{L}_{1}\left(s_{23}, s_{123}\right)\right) s_{13}\right) \\
& \mathrm{Sp}^{[\mathcal{N}=0]}\left(-P^{+} ; 1^{+}, 2^{+}, 3^{+}\right)=-\frac{[1 P][3 P]}{3\langle 12\rangle\langle 23\rangle}\left(\frac{1}{s_{123}}-\frac{\gamma_{23}^{2} \beta_{13}\left(\alpha_{32} s_{12}+\alpha_{12} s_{23}\right)}{\beta_{31} s_{12} s_{23}}\right)
\end{aligned}
$$




$$
\begin{aligned}
& \mathrm{Sp}^{[\mathcal{N}=0]}\left(-P^{+} ; 1^{-}, 2^{-}, 3^{-}\right)=\frac{1}{3} \mathrm{Sp}^{(0)}\left(-P^{+} ; 1^{-}, 2^{-}, 3^{-}\right)( \\
& \left.z_{1} z_{2}+\frac{z_{1}\left(1-z_{2}^{2}\right) z_{3} z_{2}}{\left(1-z_{1}\right)\left(1-z_{2}\right)\left(1-z_{3}\right)}+z_{3} z_{2}+z_{1} z_{3}-\frac{z_{1} z_{3}}{\gamma_{12} \gamma_{32}}\left(\frac{\gamma_{32} z_{1}}{1-z_{3}}+\frac{\gamma_{12} z_{3}}{1-z_{1}}+\frac{s_{13}}{s_{123}}\right)\right) \\
& \mathrm{Sp}^{[\mathcal{N}=0]}\left(-P^{+} ; 1^{+}, 2^{+}, 3^{-}\right)=\operatorname{Sp}^{(0)}\left(-P^{+} ; 1^{+}, 2^{+}, 3^{-}\right) \frac{\gamma_{12} s_{23}}{3 \gamma_{32}^{3}}( \\
& \frac{\gamma_{32} z_{2}}{s_{12}}\left(\frac{\gamma_{12}}{z_{3}}+\frac{\gamma_{32}}{z_{3}-1}\right)-\gamma_{12} \gamma_{32} \hat{L}_{2}\left(s_{23}, s_{123}\right) s_{23}+2 \gamma_{12} \beta_{23} \hat{L}_{3}\left(s_{23}, s_{123}\right) s_{13} s_{23} \\
& \left.+\frac{1}{s_{123}}\left(\frac{s_{23}}{s_{12}}-\frac{1}{2}\left(\gamma_{12}+2\right) \gamma_{32}\right)\right) \\
& \mathrm{Sp}^{[\mathcal{N}=0]}\left(-P^{+} ; 1^{+}, 2^{-}, 3^{+}\right)=\mathrm{Sp}^{(0)}\left(-P^{+} ; 1^{+}, 2^{-}, 3^{+}\right) \frac{\alpha_{23}^{2} s_{13}}{3 \alpha_{31}^{2}}( \\
& -\frac{2 \hat{L}_{3}\left(s_{12}, s_{123}\right) s_{13}^{2}}{\alpha_{32}}-2 \alpha_{32} \hat{L}_{3}\left(s_{23}, s_{123}\right) s_{13}^{2}-3 \hat{L}_{2}\left(s_{23}, s_{123}\right)\left(2 s_{12}+3 s_{13}\right) \\
& +\frac{5}{2}\left(\frac{2}{s_{123}}+\frac{1}{s_{23}}+\frac{1}{s_{12}}\right)-3 \hat{L}_{2}\left(s_{12}, s_{123}\right)\left(3 s_{13}+2 s_{23}\right)-\frac{1}{2 s_{123}}\left(\frac{s_{12}}{s_{23}}+\frac{s_{23}}{s_{12}}\right) \\
& \left.+\frac{s_{123}}{s_{12} s_{23}}-\frac{3 F_{\text {box }}^{1 \mathrm{~m}}}{s_{13}}\right) \\
& \mathrm{Sp}^{[\mathcal{N}=0]}\left(-P^{+} ; 1^{+}, 2^{-}, 3^{-}\right)=\frac{[1 P]^{2}}{3[23]^{2}}( \\
& \frac{2 \gamma_{23}^{2} \alpha_{21}^{2} s_{13}^{2} s_{23} \beta_{12}^{2}}{s_{12}} \hat{L}_{3}\left(s_{12}, s_{123}\right)+\frac{\gamma_{23}^{2} \alpha_{21} s_{13}\left(-s_{12}-\alpha_{21} s_{13}\right) s_{23} \beta_{12}^{2}}{s_{12} s_{123}} \hat{L}_{2}\left(s_{12}, s_{123}\right) \\
& +\frac{\gamma_{23}^{2}\left(3 \alpha_{21} s_{13}-s_{12}\right) s_{23} \beta_{12}^{2}}{s_{12} s_{123}} \hat{L}_{1}\left(s_{12}, s_{123}\right)-\frac{\gamma_{23}^{2} \alpha_{12} s_{23} \beta_{12}^{2}}{s_{12}^{2}}-\frac{\gamma_{23}^{2} \alpha_{21} s_{13} s_{23} \beta_{12}^{2}}{s_{12}^{2} s_{123}} \\
& \left.+\frac{1}{s_{123}^{2}} \frac{\gamma_{23} \alpha_{21} \beta_{12}^{2} \beta_{32} s_{13} s_{23}}{2 s_{12}}-\frac{\gamma_{23}^{2} \alpha_{21} \beta_{12}^{2} s_{13} s_{23}}{s_{12}}\right) \\
& \mathrm{Sp}^{[\mathcal{N}=0]}\left(-P^{+} ; 1^{-}, 2^{+}, 3^{-}\right)=\frac{[2 P]^{2}}{3[13]^{2}}( \\
& -2 \hat{L}_{3}\left(s_{23}, s_{123}\right) s_{123} s_{13} \gamma_{12}^{2}+\hat{L}_{2}\left(s_{23}, s_{123}\right)\left(3 \gamma_{12} s_{123}+s_{13}\right) \gamma_{12} \\
& -2 \gamma_{32}^{2} \hat{L}_{3}\left(s_{12}, s_{123}\right) s_{123} s_{13}+\frac{3\left(2 s_{12}+4 \gamma_{12} s_{123}+s_{13}\right)}{s_{123} s_{13}} \hat{L}_{0}\left(s_{23}, s_{123}\right) \\
& +\gamma_{32} \hat{L}_{2}\left(s_{12}, s_{123}\right)\left(3 \gamma_{32} s_{123}+s_{13}\right)+\hat{L}_{1}\left(s_{23}, s_{123}\right)\left(\frac{s_{13}}{s_{123}}-\frac{6 \gamma_{12}^{2} s_{123}}{s_{13}}\right) \\
& +\hat{L}_{1}\left(s_{12}, s_{123}\right)\left(\frac{s_{13}}{s_{123}}-\frac{6 \gamma_{32}^{2} s_{123}}{s_{13}}\right)+\frac{3\left(4 \gamma_{32} s_{123}+s_{13}+2 s_{23}\right)}{s_{123} s_{13}} \hat{L}_{0}\left(s_{12}, s_{123}\right) \\
& +\frac{1}{2 s_{12} s_{123} s_{23}}\left(-4 s_{123} s_{23} \gamma_{32}^{2}+\left(\gamma_{12} s_{123}\left(-3 s_{12}+2 s_{123}-3 s_{23}\right)+\left(5 s_{12}-s_{23}\right) s_{23}\right) \gamma_{32}\right. \\
& \left.\left.-\gamma_{12} s_{12}\left(s_{12}+4 \gamma_{12} s_{123}-5 s_{23}\right)\right)-\frac{3 \beta_{21} \beta_{23}}{s_{123}} F_{\text {box }}^{1 \mathrm{~m}}\right)
\end{aligned}
$$




\section{$5 \quad g \rightarrow \bar{q} q g$ splitting amplitudes}

\subsection{Colour structure and primitive amplitude decomposition}

The colour structure of the tree-level splitting amplitudes is

$$
\begin{aligned}
\mathcal{S} p^{(0)} & \left(\left\{a_{P}, \bar{\imath}_{1}, i_{2}, a_{3}\right\},-P, 1_{\bar{q}}, 2_{q}, 3\right)= \\
& T\left(a_{P}, a_{3}\right)_{i_{2}}^{\bar{q}_{1}} \mathrm{Sp}^{(0)}\left(-P ; 1_{\bar{q}}, 2_{q}, 3\right)+T\left(a_{3}, a_{P}\right)_{i_{2}}^{\bar{\tau}_{1}} \mathrm{Sp}^{(0)}\left(-P ; 2_{q}, 1_{\bar{q}}, 3\right)
\end{aligned}
$$

where $T\left(a_{1}, \ldots, a_{n}\right)_{i}^{\bar{\jmath}}=T_{\bar{\jmath} k_{1}}^{a_{1}} T_{k_{1} k_{2}}^{a_{1}} \ldots T_{k_{n-1} i}^{a_{n}}$. Note that charge conjugation symmetry allows us to write $\mathrm{Sp}^{(0)}\left(-P ; 2_{q}, 1_{\bar{q}}, 3\right)=\mathrm{Sp}^{(0)}\left(-P ; 2_{\bar{q}}, 1_{q}, 3\right)$. At one-loop we have three colour structures,

$$
\begin{aligned}
\mathcal{S} p^{(1)}\left(\left\{a_{P}, \bar{\imath}_{1}, i_{2}, a_{3}\right\},-P ; 1_{\bar{q}}, 2_{q}, 3\right)= & N_{c}\left[T\left(a_{P}, a_{3}\right)_{i_{2}}^{\bar{\tau}_{1}} \operatorname{Sp}_{4 ; 1}^{(1)}\left(-P ; 1_{\bar{q}}, 2_{q}, 3\right)\right. \\
& \left.+T\left(a_{3}, a_{P}\right)_{i_{2}}^{\bar{\imath}_{1}} \operatorname{Sp}_{4 ; 1}^{(1)}\left(-P ; 2_{q}, 1_{\bar{q}}, 3\right)\right] \\
& +\delta^{a_{P} a_{3}} \delta_{i_{2}}^{\bar{\iota}_{1}} \operatorname{Sp}_{4 ; 3}^{(1)}\left(-P ; 1_{\bar{q}}, 2_{q}, 3\right) .
\end{aligned}
$$

The partial amplitudes $\mathrm{Sp}_{4 ; 1}$ and $\mathrm{Sp}_{4 ; 3}$ are given in terms of the primitive amplitudes

$$
\begin{aligned}
\operatorname{Sp}_{4 ; 1}\left(-P ; 1_{\bar{q}}, 2_{q}, 3\right)= & \operatorname{Sp}^{[L]}\left(-P ; 1_{\bar{q}}, 2_{q}, 3\right)-\frac{1}{N_{c}^{2}} \operatorname{Sp}^{[R]}\left(-P ; 1_{\bar{q}}, 2_{q}, 3\right) \\
& +\frac{n_{f}}{N_{c}} \operatorname{Sp}^{[f]}\left(-P ; 1_{\bar{q}}, 2_{q}, 3\right) \\
\operatorname{Sp}_{4 ; 3}\left(-P ; 1_{\bar{q}}, 2_{q}, 3\right)= & \operatorname{Sp}^{[L]}\left(-P ; 1_{\bar{q}}, 2_{q}, 3\right)+\operatorname{Sp}^{[L]}\left(-P ; 2_{\bar{q}}, 1_{q}, 3\right)+\operatorname{Sp}^{[L+R]}\left(-P ; 1_{\bar{q}}, 3,2_{q}\right) \\
& +\operatorname{Sp}^{[R]}\left(-P ; 1_{\bar{q}}, 2_{q}, 3\right)+\operatorname{Sp}^{[R]}\left(-P ; 2_{\bar{q}}, 1_{q}, 3\right)
\end{aligned}
$$

where the indices $[L]$ and $[R]$ label the primitive amplitudes corresponding to fermion lines turning left or right upon entering the loop and $[f]$ denotes the primitive amplitudes with fermion-loop contribute. The label $[L+R]$ in the sub-leading colour amplitude corresponds to the sum of the left and right primitive amplitudes for the non-adjacent fermion configuration. Some representative diagrams of the primitive amplitudes are depicted in figure 3 .

The colour summed Born and virtual corrections can be written as in eq. (2.11), where the vectors $\overrightarrow{\mathrm{Sp}}^{(L)}$ and the colour matrices $\mathcal{C}_{\mathrm{Sp}}^{\left(L, L^{\prime}\right)}$ are now given by

$$
\begin{aligned}
& \overrightarrow{\mathrm{Sp}}^{(0)}=\left(\begin{array}{c}
\mathrm{Sp}^{(0)}(-P ; 1,2,3) \\
-\mathrm{Sp}^{(0)}(-P ; 2,1,3)
\end{array}\right) \\
& \mathcal{C}_{\mathrm{Sp}}^{(0,0)}=\frac{1}{N_{c}}\left(\begin{array}{cc}
N_{c}^{2}-1 & -1 \\
-1 & N_{c}^{2}-1
\end{array}\right) \\
& \overrightarrow{\mathrm{Sp}}^{(1)}=\left(\begin{array}{c}
N_{c} \operatorname{Sp}_{4 ; 1}(-P ; 1,2,3) \\
-N_{c} \operatorname{Sp}_{4 ; 1}(-P ; 2,1,3) \\
\operatorname{Sp}_{4 ; 3}(-P ; 1,2,3)
\end{array}\right) \\
& \mathcal{C}_{\mathrm{Sp}}^{(0,1)}=\frac{1}{N_{c}}\left(\begin{array}{ccc}
N_{c}^{2}-1 & -1 & N_{c} \\
-1 & N_{c}^{2}-1 & N_{c}
\end{array}\right)
\end{aligned}
$$




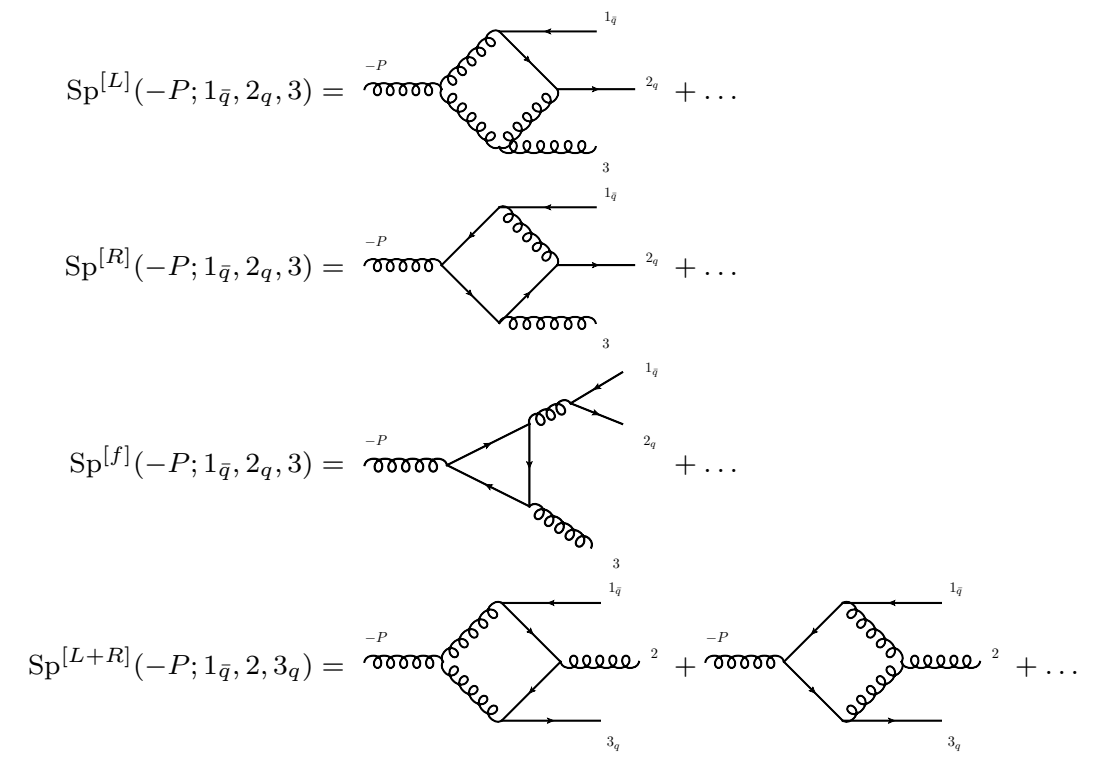

Figure 3. Sample diagrams corresponding to primitive amplitudes for $\mathrm{Sp}^{(1)}\left(-P ; 1_{\bar{q}}, 2_{q}, 3_{g}\right)$.

The quark primitive splitting amplitudes also have a useful super-symmetric decomposition [11]. In this case we can write the complicated "left-moving" amplitudes in terms of simpler ones built using the $\mathcal{N}=4$ super-multiplet,

$$
\begin{aligned}
\mathrm{Sp}^{[L]}\left(-P ; 1_{\bar{q}}, 2_{q}, 3\right)= & \mathrm{Sp}^{[\mathcal{N}=4]}\left(-P ; 1_{\bar{q}}, 2_{q}, 3\right)-\mathrm{Sp}^{[R]}\left(-P ; 1_{\bar{q}}, 2_{q}, 3\right) \\
& -\mathrm{Sp}^{[f]}\left(-P ; 1_{\bar{q}}, 2_{q}, 3\right)-\mathrm{Sp}^{[\text {scalar }]}\left(-P ; 1_{\bar{q}}, 2_{q}, 3\right) \\
\mathrm{Sp}^{[L+R]}\left(-P ; 1_{\bar{q}}, 2,3_{q}\right)= & \mathrm{Sp}^{[\mathcal{N}=4]}\left(-P ; 1_{\bar{q}}, 2,3_{q}\right)-\mathrm{Sp}^{[\text {scalar }]}\left(-P ; 1_{\bar{q}}, 2,3_{q}\right)
\end{aligned}
$$

where the $\mathrm{Sp}^{[\mathrm{scalar}]}$ function indicates the contribution from the complete scalar sector of $\mathcal{N}=4$. This includes a scalar-fermion-fermion vertex as well as the scalar-scalar-gluon vertex which contributes to the function $\mathrm{Sp}^{[\mathcal{N}=0]}$ in the pure gluonic case. Representative diagrams contributing to $\mathrm{Sp}^{[\mathrm{scalar}]}$ are shown in figure 4 . Using these relations we find a compact form for the colour dressed splitting amplitudes in terms of $\operatorname{Sp}^{[\mathcal{N}=4]}\left(-P ; 1_{\bar{q}}, 2_{q}, 3\right)$, $\mathrm{Sp}^{[R]}\left(-P ; 1_{\bar{q}}, 2_{q}, 3\right), \mathrm{Sp}^{[f]}\left(-P ; 1_{\bar{q}}, 2_{q}, 3\right), \mathrm{Sp}^{[\text {scalar }]}\left(-P ; 1_{\bar{q}}, 2_{q}, 3\right), \mathrm{Sp}^{[\mathcal{N}=4]}\left(-P ; 1_{\bar{q}}, 2,3_{q}\right)$ and $\mathrm{Sp}^{[\text {scalar] }}\left(-P ; 1_{\bar{q}}, 2,3_{q}\right)$.

\section{$5.2 \quad$ Results}

As before all results in this section are presented unrenormalized. The non-vanishing independent tree-level splitting amplitudes $g \rightarrow \bar{q} q g$ are

$$
\mathrm{Sp}^{(0)}\left(-P^{+} ; 1_{\bar{q}}^{+}, 2_{q}^{-}, 3^{-}\right)=-\frac{\left\langle z_{2}\right\rangle\left\langle z_{3}\right\rangle}{\beta_{12}[23]\langle 12\rangle}\left(\frac{\gamma_{21}}{\left(1-z_{3}\right) z_{3}}+\frac{s_{12}}{\gamma_{32} s_{123} \gamma_{21}}\right)
$$




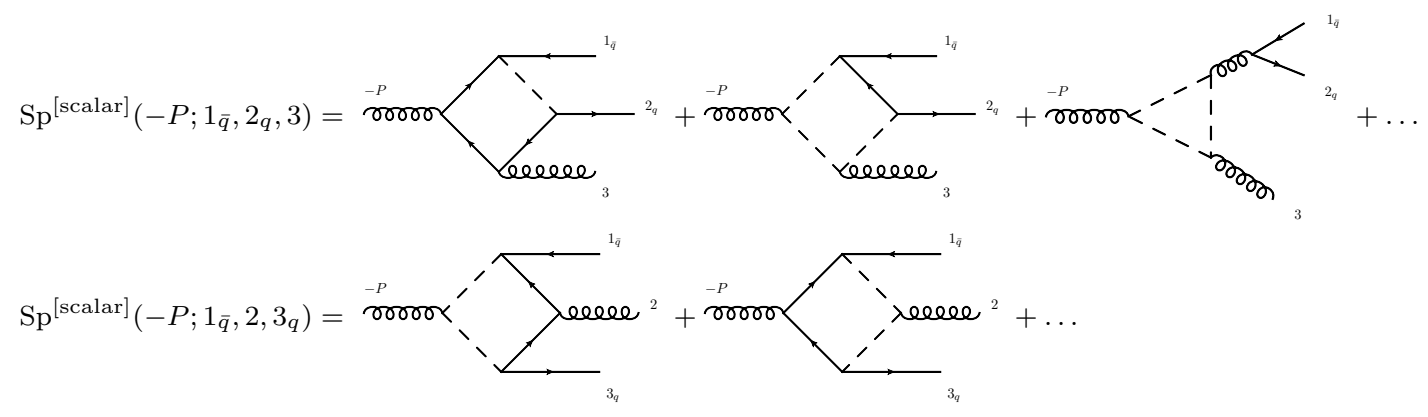

Figure 4. Sample diagrams corresponding to scalar contribution for $\mathrm{Sp}^{(1)}\left(-P ; 1_{\bar{q}}, 2_{q}, 3_{g}\right)$ in $\mathcal{N}=4$ super-symmetric Yang-Mills theory.

$$
\begin{aligned}
\mathrm{Sp}^{(0)}\left(-P^{+} ; 1_{\bar{q}}^{-}, 2_{q}^{+}, 3^{-}\right)= & -\frac{\left\langle z_{3}\right\rangle\left(\beta_{31}\right)^{\dagger}}{[13]\langle 2 P\rangle}( \\
& \left.\frac{s_{23}\left(\alpha_{13}^{2} \beta_{13}\right)^{\dagger} z_{1}^{2}}{z_{3} s_{13}\left(\beta_{31}\right)^{\dagger}}+\frac{s_{13}\left(\gamma_{31}\right)^{\dagger}}{z_{3} s_{123}}+\frac{z_{3}^{2} s_{12}\left(\alpha_{31}^{2}\right)^{\dagger}}{\left(1-z_{3}\right) s_{13}}\right) \\
\mathrm{Sp}^{(0)}\left(-P^{+} ; 1_{\bar{q}}^{+}, 2_{q}^{-}, 3^{+}\right)= & \frac{\left\langle z_{2}\right\rangle^{3}}{\left\langle z_{3}\right\rangle\langle 12\rangle\langle 23\rangle} \\
\mathrm{Sp}^{(0)}\left(-P^{+} ; 1_{\bar{q}}^{-}, 2_{q}^{+}, 3^{+}\right)= & -\frac{\left\langle z_{1}\right\rangle^{2}\left\langle z_{2}\right\rangle}{\left\langle z_{3}\right\rangle\langle 12\rangle\langle 23\rangle}
\end{aligned}
$$

and the others are obtained by conjugation using the relation

$$
\operatorname{Sp}\left(-P^{+} ; 1_{\bar{q}}^{h_{1}}, 2_{q}^{h_{2}}, 3^{h_{3}}\right)=\left.\operatorname{Sp}\left(-P^{-} ; 1_{\bar{q}}^{-h_{1}}, 2_{q}^{-h_{2}}, 3^{-h_{3}}\right)\right|_{\langle i j\rangle \leftrightarrow[i j]} .
$$

The sub-leading colour tree-level splitting amplitudes $g \rightarrow \bar{q} g q$ are not independent because they can be expressed in terms of (5.14) using the KK relation (4.4) re-written with the quark labels,

$$
\mathrm{Sp}^{(0)}\left(-P^{+} ; 1_{\bar{q}}^{h_{1}}, 2^{h_{2}}, 3_{q}^{h_{3}}\right)=-\mathrm{Sp}^{(0)}\left(-P^{+} ; 1_{\bar{q}}^{h_{1}}, 3_{q}^{h_{3}}, 2^{h_{2}}\right)+\mathrm{Sp}^{(0)}\left(-P^{+} ; 3_{\bar{q}}^{h_{3}}, 1_{q}^{h_{1}}, 2^{h_{2}}\right)
$$

A sample of two representative tree-level splitting amplitudes $g \rightarrow \bar{q} g q$ is

$$
\begin{aligned}
& \operatorname{Sp}^{(0)}\left(-P^{+} ; 1_{\bar{q}}^{+}, 2^{-}, 3_{q}^{-}\right)=\frac{[1 P]^{2}}{[12][23] s_{123}} \\
& \operatorname{Sp}^{(0)}\left(-P^{+} ; 1_{\bar{q}}^{-}, 2^{+}, 3_{q}^{+}\right)=-\frac{\left\langle z_{1}\right\rangle^{2}}{\langle 12\rangle\langle 23\rangle}
\end{aligned}
$$

The non-zero independent one-loop splitting amplitudes $g \rightarrow \bar{q} q g$ are

$$
\begin{aligned}
& \mathrm{Sp}^{[\mathcal{N}=4]}\left(-P^{+} ; 1_{\bar{q}}^{+}, 2_{q}^{-}, 3^{-}\right)=\mathrm{Sp}^{(0)}\left(-P^{+} ; 1_{\bar{q}}^{+}, 2_{q}^{-}, 3^{-}\right) V_{g} \\
& \quad-\frac{\left\langle z_{2}\right\rangle\left\langle z_{3}\right\rangle}{[23]\langle 12\rangle}\left(\frac{\left(1-z_{1}\right)^{2} s_{12}}{\gamma_{21} z_{3} s_{1 P}} \mathrm{~F}_{2}^{\mathrm{NMHV}}-\left(\frac{\gamma_{32}^{2} s_{2 P}^{2}\left(\gamma_{12}^{2}\right)^{\dagger}}{\left(1-z_{3}\right) \gamma_{23} s_{12} s_{3 P}}-\frac{\gamma_{23}}{\left(1-z_{3}\right) z_{3}}\right) \mathrm{F}_{1}^{\mathrm{NMHV}}\right.
\end{aligned}
$$




$$
\begin{aligned}
& -\frac{z_{3} s_{12} s_{13}^{3}}{\gamma_{21} \gamma_{23} \gamma_{31}^{2} s_{123} s_{1 P}^{2} s_{3 P}\left(\gamma_{31}\right)^{\dagger}}\left(\frac{z_{1} \beta_{12} s_{23}^{3}}{s_{12} s_{13} s_{3 P}\left(\gamma_{13}\right)^{\dagger}}-\frac{\gamma_{31}^{2} s_{1 P}\left(\gamma_{31}\right)^{\dagger} s_{23}^{2}}{z_{3} s_{13}^{3}}\right. \\
& \left.\left.+\frac{z_{1} \beta_{32} s_{1 P}}{s_{13}\left(\gamma_{13}\right)^{\dagger}}+\frac{\gamma_{23} z_{3} s_{3 P}}{\gamma_{21} s_{13}\left(\gamma_{31}\right)^{\dagger}}\right) \mathrm{F}_{3}^{\mathrm{NMHV}}\right) \\
& \mathrm{Sp}^{[\mathcal{N}=4]}\left(-P^{+} ; 1_{\bar{q}}^{-}, 2_{q}^{+}, 3^{-}\right)=\mathrm{Sp}^{(0)}\left(-P^{+} ; 1_{\bar{q}}^{-}, 2_{q}^{+}, 3^{-}\right) V_{g} \\
& -\frac{\left\langle z_{3}\right\rangle[2 P]}{[13]}\left(-\frac{\gamma_{32} z_{2} s_{3 P}\left(\gamma_{23}\right)^{\dagger}\left(\gamma_{31}\right)^{\dagger} \gamma_{13}^{2}}{\gamma_{23} z_{3}^{2} s_{13} s_{23}} \mathrm{~F}_{2}^{\mathrm{NMHV}}\right. \\
& +\frac{\gamma_{32}}{\left(1-z_{3}\right) \gamma_{23} s_{3 P}}\left(\frac{\gamma_{31} z_{2}^{2}}{\gamma_{21}}-\frac{\gamma_{13} \gamma_{21} z_{1} s_{13}}{\gamma_{31} z_{3} s_{12}\left(\gamma_{13}\right)^{\dagger}}\right) \mathrm{F}_{1}^{\mathrm{NMHV}} \\
& \left.-\left(\frac{\gamma_{12} s_{13}\left(\gamma_{31}\right)^{\dagger}}{z_{3} s_{12} s_{3 P}}+\frac{s_{13}\left(\gamma_{31}\right)^{\dagger}}{z_{3} s_{123} s_{3 P}}+\frac{z_{1} s_{13}}{\gamma_{12} \gamma_{32} s_{123} s_{3 P}\left(\gamma_{13}\right)^{\dagger}}\right) \mathrm{F}_{3}^{\mathrm{NMHV}}\right) \\
& \mathrm{Sp}^{[\mathcal{N}=4]}\left(-P^{+} ; 1_{\bar{q}}^{+}, 2_{q}^{-}, 3^{+}\right)=\mathrm{Sp}^{(0)}\left(-P^{+} ; 1_{\bar{q}}^{+}, 2_{q}^{-}, 3^{+}\right)\left(V_{g}+F^{\mathrm{MHV}}\right) \\
& \mathrm{Sp}^{[\mathcal{N}=4]}\left(-P^{+} ; 1_{\bar{q}}^{-}, 2_{q}^{+}, 3^{+}\right)=\mathrm{Sp}^{(0)}\left(-P^{+} ; 1_{\bar{q}}^{-}, 2_{q}^{+}, 3^{+}\right)\left(V_{g}+F^{\mathrm{MHV}}\right) \\
& \mathrm{Sp}^{[\mathrm{R}]}\left(-P^{+} ; 1_{\bar{q}}^{+}, 2_{q}^{-}, 3^{-}\right)=\mathrm{Sp}^{(0)}\left(-P^{+} ; 1_{\bar{q}}^{+}, 2_{q}^{-}, 3^{-}\right)\left(-\frac{1}{\epsilon^{2}}\left(\frac{\mu_{R}}{-s_{12}}\right)^{\epsilon}-\frac{3}{2 \epsilon}\left(\frac{\mu_{R}}{-s_{12}}\right)^{\epsilon}\right. \\
& \left.-\frac{7}{2}-\frac{\delta_{\mathrm{R}}}{2}\right)-\frac{\alpha_{21}\left\langle z_{2}\right\rangle\left\langle z_{3}\right\rangle}{[23]\langle 12\rangle}\left(-\frac{s_{12}}{2 \gamma_{21} \gamma_{31} \alpha_{21} s_{123}} \mathrm{~F}_{\text {box }}^{1 m}-\frac{1}{2} s_{123} s_{23} \hat{L}_{2}\left(s_{12}, s_{123}\right)\right. \\
& \left.+\frac{1}{2}\left(-3 s_{123}-\frac{2 s_{23}}{\gamma_{32}}\right) \hat{L}_{1}\left(s_{12}, s_{123}\right)+\frac{1}{2}+\frac{s_{23}}{4 s_{12}}\right) \\
& \mathrm{Sp}^{[\mathrm{R}]}\left(-P^{+} ; 1_{\bar{q}}, 2_{q}^{+}, 3^{-}\right)=\mathrm{Sp}^{(0)}\left(-P^{+} ; 1_{\bar{q}}^{-}, 2_{q}^{+}, 3^{-}\right)\left(-\frac{1}{\epsilon^{2}}\left(\frac{\mu_{R}}{-s_{12}}\right)^{\epsilon}-\frac{3}{2 \epsilon}\left(\frac{\mu_{R}}{-s_{12}}\right)^{\epsilon}\right. \\
& \left.-\frac{7}{2}-\frac{\delta_{\mathrm{R}}}{2}\right)+\frac{\left\langle z_{3}\right\rangle[2 P]}{[13] s_{13}}\left(\frac{\beta_{12} \beta_{21} s_{13}}{2 \gamma_{31} s_{123}} \mathrm{~F}_{\text {box }}^{1 m}-\frac{\beta_{12} \beta_{21} s_{13}^{2} s_{23}}{2 \gamma_{31} s_{123}} \hat{L}_{2}\left(s_{12}, s_{123}\right)\right. \\
& -\frac{3 \beta_{12} \beta_{21} s_{13}^{2}}{2 \gamma_{31} s_{123}} \hat{L}_{1}\left(s_{12}, s_{123}\right)+\frac{s_{12} s_{23} \gamma_{12}^{2}}{2 \gamma_{32}} \hat{L}_{2}\left(s_{23}, s_{123}\right) \\
& +\frac{s_{12} \gamma_{12}}{\gamma_{32}}\left(\frac{\gamma_{12}\left(2 s_{12} s_{123}+3 s_{23} s_{123}-3 s_{12} s_{23}\right)}{2 s_{12} s_{123}}+1\right) \hat{L}_{1}\left(s_{23}, s_{123}\right) \\
& +\frac{1}{2}\left(3 \gamma_{12}-\frac{s_{23}}{\gamma_{32} s_{123}}-\frac{3\left(\gamma_{12}-1\right) s_{12}}{s_{123}}-1\right) \hat{L}_{0}\left(s_{23}, s_{123}\right) \\
& \left.-\frac{\gamma_{12}^{2} s_{23}}{4 \gamma_{32} s_{12}}+\frac{\gamma_{12} s_{23}}{2 \gamma_{32} s_{123}}+\gamma_{12}+\frac{\left(1-\gamma_{12}\right) s_{12}}{4 s_{123}}-\frac{\left(s_{123}-s_{23}\right) s_{12}}{4 \gamma_{32} s_{123}^{2}}\right)
\end{aligned}
$$




$$
\begin{aligned}
& \mathrm{Sp}^{[\mathrm{R}]}\left(-P^{+} ; 1_{\bar{q}}^{+}, 2_{q}^{-}, 3^{+}\right)=\mathrm{Sp}^{(0)}\left(-P^{+} ; 1_{\bar{q}}^{+}, 2_{q}^{-}, 3^{+}\right)\left(-\frac{1}{\epsilon^{2}}\left(\frac{\mu_{R}}{-s_{12}}\right)^{\epsilon}-\frac{3}{2 \epsilon}\left(\frac{\mu_{R}}{-s_{12}}\right)^{\epsilon}\right. \\
& \quad-\frac{7}{2}-\frac{\delta_{\mathrm{R}}}{2}-\frac{\alpha_{12}^{3}}{\alpha_{13}^{3}}\left(-\frac{\mathrm{F}_{\text {box }}^{1 m}}{2}-\frac{1}{2}\left(6 s_{12}^{2}+s_{23}^{2}-6 s_{12} s_{123}\right) \hat{L}_{2}\left(s_{12}, s_{123}\right)\right. \\
& +\left(3 s_{123}-2 s_{23}\right) \hat{L}_{1}\left(s_{12}, s_{123}\right)+\frac{3}{2} \hat{L}_{0}\left(s_{12}, s_{123}\right) \\
& +\frac{\left(s_{123}^{2}-s_{12}^{2}-s_{23}^{2}\right)}{2 \alpha_{12}^{2}} \hat{L}_{2}\left(s_{23}, s_{123}\right)+\left(\frac{s_{23}}{\alpha_{12}^{2}}-\frac{2 s_{13}}{\alpha_{12}}\right) \hat{L}_{1}\left(s_{23}, s_{123}\right) \\
& \left.\left.\quad-\frac{\left(s_{123}+s_{23}\right)^{2}-s_{12}^{2}-2 s_{23} s_{12}}{4 \alpha_{12}^{2} s_{123} s_{23}}-\frac{6 s_{12}^{2}-s_{23}^{2}-2 s_{23} s_{12}}{4 s_{123} s_{12}}+\frac{s_{13}}{\alpha_{12} s_{123}}-\frac{3}{2}\right)\right)
\end{aligned}
$$

$$
\begin{aligned}
\mathrm{Sp}^{[\mathrm{R}]} & \left(-P^{+} ; 1_{\bar{q}}^{-}, 2_{q}^{+}, 3^{+}\right)=\mathrm{Sp}^{(0)}\left(-P^{+} ; 1_{\bar{q}}^{-}, 2_{q}^{+}, 3^{+}\right)\left(-\frac{1}{\epsilon^{2}}\left(\frac{\mu_{R}}{-s_{12}}\right)^{\epsilon}-\frac{3}{2 \epsilon}\left(\frac{\mu_{R}}{-s_{12}}\right)^{\epsilon}\right. \\
& -\frac{7}{2}-\frac{\delta_{\mathrm{R}}}{2}+\frac{\alpha_{12}}{\alpha_{13}}\left(\frac{\mathrm{F}_{\text {box }}^{1 m}}{2}+\frac{\alpha_{12}^{2} s_{23}^{2}}{2} \hat{L}_{2}\left(s_{12}, s_{123}\right)-2 \alpha_{12} s_{23} \hat{L}_{1}\left(s_{12}, s_{123}\right)\right. \\
& \left.\left.+\frac{3}{2} \hat{L}_{0}\left(s_{12}, s_{123}\right)+\frac{\left(s_{123}-s_{12}\right)}{2 s_{123}}+\frac{\alpha_{12} s_{23}}{s_{123}}-\frac{\alpha_{12}^{2} s_{23}^{2}}{4 s_{12} s_{123}}\right)\right)
\end{aligned}
$$

$$
\begin{aligned}
& \operatorname{Sp}^{[\mathrm{f}]}\left(-P^{+} ; 1_{\bar{q}}^{+}, 2_{q}^{-}, 3^{-}\right)=\mathrm{Sp}^{(0)}\left(-P^{+} ; 1_{\bar{q}}^{+}, 2_{q}^{-}, 3^{-}\right)\left(-\frac{2}{3 \epsilon}\left(\frac{\mu_{R}}{-s_{12}}\right)^{\epsilon}-\frac{10}{9}\right) \\
& -\frac{\left\langle z_{2}\right\rangle\left\langle z_{3}\right\rangle}{3[12]\langle 23\rangle}\left(-\frac{2 s_{23}}{\gamma_{23}} \hat{L}_{3}\left(s_{12}, s_{123}\right)-\frac{1}{\gamma_{21}} \hat{L}_{2}\left(s_{12}, s_{123}\right)\right.
\end{aligned}
$$$$
\left.-\frac{2}{\gamma_{21} \gamma_{31} s_{123}} \hat{L}_{1}\left(s_{12}, s_{123}\right)-\frac{2}{\gamma_{21} \gamma_{31} s_{123} s_{23}} \hat{L}_{0}\left(s_{12}, s_{123}\right)+\frac{1}{2 \gamma_{21} s_{12} s_{123}}\right)
$$

$$
\begin{aligned}
\mathrm{Sp}^{[\mathrm{f}]} & \left(-P^{+} ; 1_{\bar{q}}^{-}, 2_{q}^{+}, 3^{-}\right)=\mathrm{Sp}^{(0)}\left(-P^{+} ; 1_{\bar{q}}^{-}, 2_{q}^{+}, 3^{-}\right)\left(-\frac{2}{3 \epsilon}\left(\frac{\mu_{R}}{-s_{12}}\right)^{\epsilon}-\frac{10}{9}\right) \\
& +\frac{\left\langle z_{3}\right\rangle s_{13}[2 P]}{3[13]}\left(2 \gamma_{32} s_{123} \hat{L}_{3}\left(s_{12}, s_{123}\right)-\hat{L}_{2}\left(s_{12}, s_{123}\right)\right. \\
& +\left(\frac{2 \gamma_{12}^{2}}{\gamma_{32} s_{123}}+\frac{2 s_{123} \gamma_{12}}{s_{12}^{2}}-\frac{2}{s_{12}}\right) \hat{L}_{1}\left(s_{12}, s_{123}\right) \\
& \left.+\left(\frac{2 \gamma_{12}\left(s_{12}+s_{123}\right)}{s_{12}^{2} s_{123}}-\frac{2}{s_{12} s_{123}}\right) \hat{L}_{0}\left(s_{12}, s_{123}\right)-\frac{1}{2 s_{12} s_{123}}\right)
\end{aligned}
$$$$
\mathrm{Sp}^{[\mathrm{f}]}\left(-P^{+} ; 1_{\bar{q}}^{+}, 2_{q}^{-}, 3^{+}\right)=\mathrm{Sp}^{(0)}\left(-P^{+} ; 1_{\bar{q}}^{+}, 2_{q}^{-}, 3^{+}\right)\left(-\frac{2}{3 \epsilon}\left(\frac{\mu_{R}}{-s_{12}}\right)^{\epsilon}-\frac{10}{9}\right.
$$$$
-\frac{\alpha_{12}^{3}}{3 \alpha_{13}^{3}}\left(\left(s_{13}^{3}-s_{23}^{3}\right) \hat{L}_{3}\left(s_{12}, s_{123}\right)-\frac{3\left(s_{13}^{2}+2 s_{23} s_{13}\right)}{\alpha_{12}} \hat{L}_{2}\left(s_{12}, s_{123}\right)\right.
$$ 


$$
\begin{aligned}
& +\frac{3 s_{13}}{\alpha_{12}^{2}} \hat{L}_{1}\left(s_{12}, s_{123}\right)-\hat{L}_{0}\left(s_{12}, s_{123}\right)-\frac{1}{2} \frac{\left(s_{123}-s_{13}\right)^{2}-s_{13} s_{123}-s_{12}\left(s_{123}-s_{23}\right)}{s_{12} s_{123}} \\
& \left.\left.-\frac{s_{13}}{\alpha_{12}^{2} s_{123}}+\frac{s_{13}\left(3 s_{12}+5 s_{123}+s_{23}\right)}{2 \alpha_{12} s_{12} s_{123}}\right)\right)
\end{aligned}
$$

$$
\begin{aligned}
& \operatorname{Sp}^{[\mathrm{f}]}\left(-P^{+} ; 1_{\bar{q}}^{-}, 2_{q}^{+}, 3^{+}\right)=\mathrm{Sp}^{(0)}\left(-P^{+} ; 1_{\bar{q}}^{-}, 2_{q}^{+}, 3^{+}\right)\left(-\frac{2}{3 \epsilon}\left(\frac{\mu_{R}}{-s_{12}}\right)^{\epsilon}-\frac{10}{9}\right. \\
& -\frac{\alpha_{12}}{3 \alpha_{13}}\left(-2 \alpha_{12}^{2} s_{23}^{3} \hat{L}_{3}\left(s_{12}, s_{123}\right)+3 \alpha_{12} s_{23}^{2} \hat{L}_{2}\left(s_{12}, s_{123}\right)+2 s_{13} \hat{L}_{1}\left(s_{12}, s_{123}\right)\right. \\
& \left.\left.\quad-\frac{s_{23}}{s_{12}-s_{123}} \hat{L}_{0}\left(s_{12}, s_{123}\right)+\frac{s_{23}}{s_{123}}-\frac{\alpha_{12} s_{23}^{2}}{2 s_{12} s_{123}}\right)\right)
\end{aligned}
$$

$$
\begin{aligned}
& \mathrm{Sp}^{\text {[scalar] }}\left(-P^{+} ; 1_{\bar{q}}^{+}, 2_{q}^{-}, 3^{-}\right)=\mathrm{Sp}^{(0)}\left(-P^{+} ; 1_{\bar{q}}^{+}, 2_{q}^{-}, 3^{-}\right)\left(\frac{2}{3} \delta_{\mathrm{R}}\right) \\
& \quad+\frac{3\left\langle z_{2}\right\rangle\left\langle z_{3}\right\rangle}{\gamma_{21} \gamma_{32}[12]\langle 23\rangle s_{123}} \hat{L}_{1}\left(s_{12}, s_{123}\right)
\end{aligned}
$$

$$
\begin{aligned}
& \mathrm{Sp}^{[\text {scalar }]}\left(-P^{+} ; 1_{\bar{q}}^{-}, 2_{q}^{+}, 3^{-}\right)=\mathrm{Sp}^{(0)}\left(-P^{+} ; 1_{\bar{q}}^{-}, 2_{q}^{+}, 3^{-}\right)\left(\frac{2}{3} \delta_{\mathrm{R}}-2\right) \\
& \frac{\left\langle z_{3}\right\rangle}{[13]\langle 2 P\rangle}\left(+\frac{3 s_{2 P}}{2 \gamma_{31} s_{123}} \mathrm{~F}_{\text {box }}^{1 m}-\frac{3 s_{13} s_{2 P}}{\gamma_{31} s_{123}} \hat{L}_{1}\left(s_{12}, s_{123}\right)-\frac{3 s_{13} s_{2 P}}{\gamma_{31} s_{123}} \hat{L}_{1}\left(s_{23}, s_{123}\right)\right. \\
& \left.\quad+\frac{2 s_{13} s_{2 P}}{\gamma_{32} s_{12} s_{123}}-\frac{2 \gamma_{13} \gamma_{32} z_{2} s_{2 P}}{\gamma_{23}\left(1-z_{3}\right) s_{12}}+\frac{2 \gamma_{12} \gamma_{13} z_{2} s_{2 P}}{\gamma_{23} z_{3} s_{12}}\right)
\end{aligned}
$$$$
\mathrm{Sp}^{[\text {scalar }]}\left(-P^{+} ; 1_{\bar{q}}^{+}, 2_{q}^{-}, 3^{+}\right)=\mathrm{Sp}^{(0)}\left(-P^{+} ; 1_{\bar{q}}^{+}, 2_{q}^{-}, 3^{+}\right)\left(\frac{2}{3} \delta_{\mathrm{R}}-\frac{3 \alpha_{12}}{\alpha_{13}^{2}}\left(\frac{1}{2} \mathrm{~F}_{\text {box }}^{1 m}\right.\right.
$$$$
\left.\left.+s_{12} \hat{L}_{1}\left(s_{23}, s_{123}\right)+s_{23} \hat{L}_{1}\left(s_{12}, s_{123}\right)+\hat{L}_{0}\left(s_{12}, s_{123}\right)+\hat{L}_{0}\left(s_{23}, s_{123}\right)\right)\right)
$$

$$
\mathrm{Sp}^{[\text {scalar }]}\left(-P^{+} ; 1_{\bar{q}}^{-}, 2_{q}^{+}, 3^{+}\right)=\mathrm{Sp}^{(0)}\left(-P^{+} ; 1_{\bar{q}}^{-}, 2_{q}^{+}, 3^{+}\right)\left(\frac{2}{3} \delta_{\mathrm{R}}-3 \alpha_{12} s_{23} \hat{L}_{1}\left(s_{12}, s_{123}\right)\right)
$$

The expressions for the non-zero independent one loop splitting amplitudes $g \rightarrow \bar{q} g q$ are

$$
\begin{aligned}
& \mathrm{Sp}^{[\mathcal{N}=4]}\left(-P^{+} ; 1_{\bar{q}}^{+}, 2^{-}, 3_{q}^{-}\right)=\mathrm{Sp}^{(0)}\left(-P^{+} ; 1_{\bar{q}}^{+}, 2^{-}, 3_{q}^{-}\right)\left(V_{g}+\frac{\gamma_{21}\left(1-z_{1}\right)^{2} s_{123}}{z_{2} s_{1 P}} \mathrm{~F}_{2}^{\mathrm{NMHV}}\right. \\
& \left.\quad+\left(\frac{\gamma_{21} \alpha_{23} s_{23}\left(\gamma_{13}\right)^{\dagger}}{z_{1} s_{1 P}}+1\right) \mathrm{F}_{3}^{\mathrm{NMHV}}-\frac{\gamma_{31}^{2} s_{123}\left(\gamma_{13} \alpha_{23}\right)^{\dagger}}{s_{13}} \mathrm{~F}_{1}^{\mathrm{NMHV}}\right)
\end{aligned}
$$




$$
\begin{aligned}
& \operatorname{Sp}^{[\mathcal{N}=4]}\left(-P^{+} ; 1_{\bar{q}}^{-}, 2^{+}, 3_{q}^{+}\right)=\operatorname{Sp}^{(0)}\left(-P^{+} ; 1_{\bar{q}}^{-}, 2^{+}, 3_{q}^{+}\right)\left(V_{g}+F^{\mathrm{MHV}}\right) \\
& \mathrm{Sp}^{[\text {scalar }]}\left(-P^{+} ; 1_{\bar{q}}^{+}, 2^{-}, 3_{q}^{-}\right)=\mathrm{Sp}^{(0)}\left(-P^{+} ; 1_{\bar{q}}^{+}, 2^{-}, 3_{q}^{-}\right)\left(-\frac{2}{3 \epsilon}\left(\frac{\mu_{R}}{-s_{13}}\right)^{\epsilon}-\frac{10}{9}\right. \\
& \left.\quad+\frac{2 \delta_{\mathrm{R}}}{3}+\frac{3 \gamma_{21} s_{23}}{\gamma_{23}} \hat{L}_{1}\left(s_{12}, s_{123}\right)-\frac{2}{3} \hat{L}_{0}\left(s_{13}, s_{123}\right)\right) \\
& \mathrm{Sp}^{[\mathrm{scalar}]}\left(-P^{+} ; 1_{\bar{q}}^{-}, 2^{+}, 3_{q}^{+}\right)=\mathrm{Sp}^{(0)}\left(-P^{+} ; 1_{\bar{q}}^{-}, 2^{+}, 3_{q}^{+}\right)\left(-\frac{2}{3 \epsilon}\left(\frac{\mu_{R}}{-s_{123}}\right)^{\epsilon}-\frac{29}{18}\right. \\
& \quad+\frac{2 \delta_{\mathrm{R}}}{3}+\left(\frac{\gamma_{32}^{2} s_{12}^{2}}{2 \gamma_{12}^{2}}-\frac{s_{13}^{2}}{2 \gamma_{13}^{2}}\right) \hat{L}_{2}\left(s_{12}, s_{123}\right)-4 \alpha_{12} s_{23} \hat{L}_{1}\left(s_{12}, s_{123}\right) \\
& \left.\quad+\frac{1}{2} \hat{L}_{0}\left(s_{12}, s_{123}\right)+\frac{\gamma_{32}^{2} s_{12}}{4 \gamma_{12}^{2} s_{123}}-\frac{\gamma_{32}}{\gamma_{12}}-\frac{s_{13}^{2}}{4 \gamma_{13}^{2} s_{12} s_{123}}+\frac{s_{123}}{2 s_{12}}\right)
\end{aligned}
$$

The expressions for the others helicity configurations are obtained by conjugation operation or by reverting the fermion line, namely $\operatorname{Sp}\left(-P ; 1_{\bar{q}}^{h}, 2,3_{q}^{-h}\right)=\operatorname{Sp}\left(-P ; 3_{\bar{q}}^{-h}, 2,1_{q}^{h}\right)$.

\section{Super-symmetric Ward identities}

Super-symmetric Ward identities (SWI) allow us to relate amplitudes with different particle content in super-symmetric theories $[67,68]$. We have checked that the super-symmetric primitive amplitudes presented in the previous sections obey the expected SWI which are easy to derive by taking the triple collinear limit of the equations presented [68]. The resulting equations differ for the MHV and NMHV helicity configurations,

$$
\begin{aligned}
\operatorname{Sp}^{[\mathcal{N}=4]}\left(-P^{+} ; 1^{+}, 2^{-}, 3^{+}\right) & -\frac{\left\langle z_{2}\right\rangle}{\left\langle z_{1}\right\rangle} \operatorname{Sp}^{[\mathcal{N}=4]}\left(-P^{+} ; 1_{\bar{q}}^{+}, 2_{q}^{-}, 3^{+}\right)=0, \\
\operatorname{Sp}^{[\mathcal{N}=4]}\left(-P^{+} ; 1^{-}, 2^{+}, 3^{+}\right) & +\frac{\left\langle z_{1}\right\rangle}{\left\langle z_{2}\right\rangle} \operatorname{Sp}^{[\mathcal{N}=4]}\left(-P^{+} ; 1_{\bar{q}}^{-}, 2_{q}^{+}, 3^{+}\right)=0, \\
\operatorname{Sp}^{[\mathcal{N}=4]}\left(-P^{+} ; 1^{+}, 2^{-}, 3^{-}\right) & -\frac{\left\langle z_{2}\right\rangle}{\left\langle z_{1}\right\rangle} \operatorname{Sp}^{[\mathcal{N}=4]}\left(-P^{+} ; 1_{\bar{q}}^{+}, 2_{q}^{-}, 3^{-}\right) \\
& -\frac{\left\langle z_{3}\right\rangle}{\left\langle z_{1}\right\rangle} \operatorname{Sp}^{[\mathcal{N}=4]}\left(-P^{+} ; 1_{\bar{q}}^{+}, 2^{-}, 3_{q}^{-}\right)=0, \\
\mathrm{Sp}^{[\mathcal{N}=4]}\left(-P^{+} ; 1^{-}, 2^{+}, 3^{-}\right) & +\frac{\left\langle z_{1}\right\rangle}{\left\langle z_{2}\right\rangle} \operatorname{Sp}^{[\mathcal{N}=4]}\left(-P^{+} ; 1_{\bar{q}}^{-}, 2_{q}^{+}, 3^{-}\right) \\
& +\frac{\left\langle z_{3}\right\rangle}{\left\langle z_{2}\right\rangle} \operatorname{Sp}^{[\mathcal{N}=4]}\left(-P^{+} ; 3_{\bar{q}}^{-}, 2_{q}^{+}, 1^{-}\right)=0 .
\end{aligned}
$$

The SWI are also applicable to tree-level amplitudes in non-super-symmetric theories. 


\section{Cross checks}

We check the universality of the splitting functions derived in the previous section numerically against the six parton amplitudes available in NJET [69]. In order to make sure we could evaluate as close to the precise limit as possible, we implemented the checks in octuple precision using the QD and ONELOOP [70] packages.

We check the validity of eq. (2.9) by computing the ratio between the two sides of the equation summed over the external helicities

$$
\begin{aligned}
& r_{\text {collinear123 }}= \\
& \frac{\sum_{\lambda_{i}} \mathcal{M}_{n}^{\left(L, L^{\prime}\right)}\left(\left\{p_{i}^{\lambda_{i}}\right\}\right)}{\sum_{k=0}^{L} \sum_{k^{\prime}=0}^{L^{\prime}} \sum_{\lambda_{P}, \lambda_{P}^{\prime}} \mathcal{P}_{m, s ;-\lambda_{P},-\lambda_{P}^{\prime}}^{\left(L-k, L^{\prime}\right.}\left(-P ;\left\{p_{i}\right\}_{i=1}^{m}\right) \mathcal{M}_{n-m+1, s ; \lambda_{P}, \lambda_{P}^{\prime}}^{\left(k, k^{\prime}\right)}\left(\left\{P,\left\{p_{i}\right\}_{i=m+1}^{n}\right\}\right)}
\end{aligned}
$$

where $\mathcal{M}_{n, s}$ and $\mathcal{P}_{n, s}$ in the denominator are defined from $\mathcal{M}_{n}$ and $\mathcal{P}_{n}$ by summing over the external helicities:

$$
\begin{aligned}
\mathcal{M}_{n-m+1 ; \lambda_{P}, \lambda_{P}^{\prime}}^{\left(L, L^{\prime}\right)}\left(P,\left\{p_{i}\right\}\right) & =\sum_{\lambda_{m+1}, \ldots, \lambda_{n}} \mathcal{M}_{n-m+1, s ; \lambda_{P}, \lambda_{P}^{\prime}}^{\left(L, L^{\prime}\right)}\left(P,\left\{p_{i}^{\lambda_{i}}\right\}\right) \\
\mathcal{P}_{m ; \lambda_{P}, \lambda_{P}^{\prime}}^{\left(L, L^{\prime}\right)}\left(-P ;\left\{p_{i}\right\}\right) & =\sum_{\lambda_{1}, \ldots, \lambda_{m}} \mathcal{P}_{m, s ; \lambda_{P}, \lambda_{P}^{\prime}}^{\left(L, L^{\prime}\right)}\left(-P ;\left\{p_{i}^{\lambda_{i}}\right\}\right) .
\end{aligned}
$$

Eq. (2.9) obviously implies

$$
r_{\text {collinear123 }} \stackrel{1 \| 2|| 3}{\longrightarrow} 1 .
$$

It is worth observing that the finite one-loop all-plus and all-minus four-gluon helicity amplitudes, while giving no contribution to the NLO squared matrix element, they give instead a finite contribution to $r_{\text {collinear123 }}$ because of spin correlations.

In figure 5 we plot $r_{\text {collinear123 }}-1$ as a function of the invariant mass $s_{123}$ of the three collinear partons. More in detail we verify the validity of eq. (7.2) in double, doubledouble and double-quadruple precision for both gluon (on the left) and quark (on the right) splitting functions. As one can see, going to higher precision allowed us to make stronger checks on phase-space space points which are closer to the limit, where the numerical evaluation is highly unstable at lower floating-point precision.

Similarly, we also numerically verified eq. (2.8) for each primitive amplitude and all the helicity configurations, although all of these already contribute to the check described above.

As well as the numerical checks we have also verified that all splitting functions factorise correctly in the iterated collinear limit,

$$
\begin{aligned}
\lim _{1 \| 2} \mathrm{Sp}^{(L)} & \left(-P_{123}^{-\lambda_{P}}, 1^{\lambda_{1}}, 2^{\lambda_{2}}, 3^{\lambda_{3}}\right) \\
& =\sum_{\lambda= \pm} \sum_{k=0}^{L} \mathrm{Sp}^{(L-k)}\left(-P_{\widetilde{123}^{-\lambda_{P}}}, \tilde{P}_{12}^{\lambda}, 3^{\lambda_{3}}\right) \mathrm{Sp}^{(k)}\left(-P_{12}^{-\lambda}, 1^{\lambda_{1}}, 2^{\lambda_{2}}\right),
\end{aligned}
$$

where the scale $s_{12} \ll s_{123}$ and $P_{\widetilde{123}}=\tilde{P}_{12}+p_{3}$. All di-logarithms drop out in this limit though some care should be taken to ensure the hierarchy of scales is imposed correctly. 

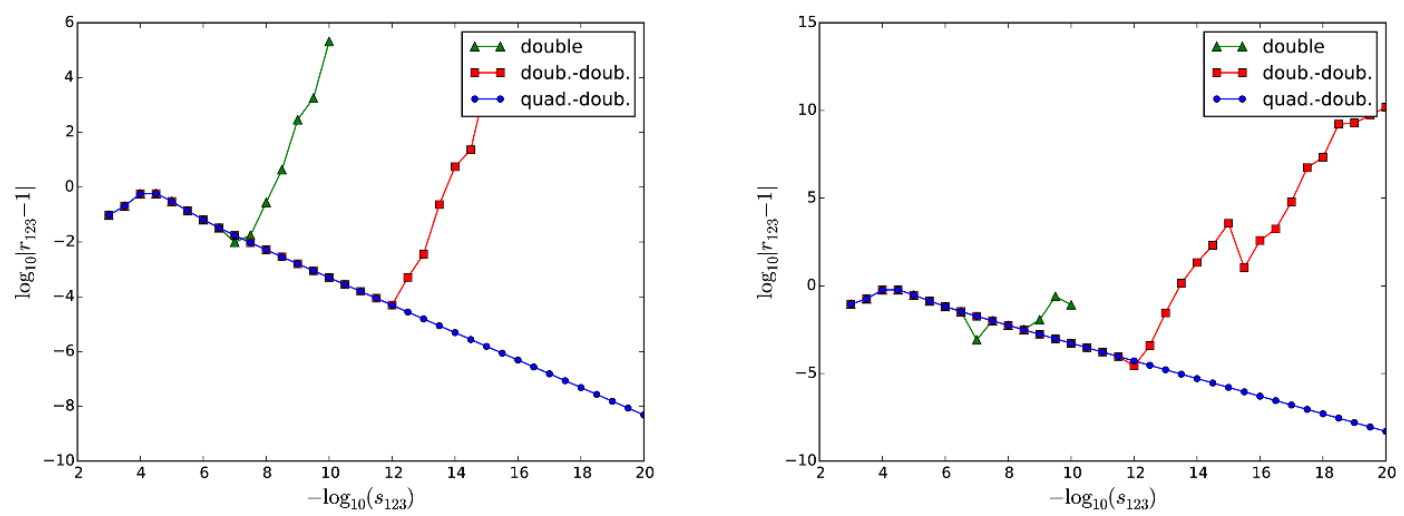

Figure 5. Numerical check of the collinear limit of $r_{\text {collinear123 }}-1$, with $r_{\text {collinear123 defined by }}$ eq. (7.1), as a function of the invariant mass $s_{123}$, from $s_{123}=10^{-3}$ to $s_{123}=10^{-20}$. For this check we set $n_{f}=5, \sqrt{s}=10^{3}$ and $\mu_{R}=10^{3} / 7$. The plot on the left shows the all-gluon case, while the one on the right shows the quark case. In the latter it was not possible to obtain numerical results in double precision for $s_{123} \lesssim 10^{-10}$.

\section{Conclusions}

In this article we have computed the one-loop triple collinear splitting amplitudes in QCD initiated by a gluon. These functions are one of the last remaining ingredients to complete the classification of universal infrared limits relevant at $\mathrm{N}^{3} \mathrm{LO}$.

Some effort has been taken to ensure the splitting amplitudes have compact analytic forms. We made use of the spinor-helicity formalism and super-symmetric decompositions and related the pure gluonic amplitudes to the ones containing a quark anti-quark pair. The primitive amplitude colour decomposition was also a useful tool to express full colour and helicity summed splitting functions which were all checked explicitly against the numerical matrix elements for $2 \rightarrow 4$ scattering in NJET. In the course of these checks we made use of the high precision numerical evaluation available with up to 64 digits via the QD package. This allowed us to probe deep into the collinear limit and verify that all parts of the computation behaved correctly. This was particularly important for the spin correlated and sub-leading colour corrections which are significantly suppressed.

There are still some missing ingredients needed for the constructions of a fully differential $\mathrm{N}^{3} \mathrm{LO}$ subtraction scheme. Firstly, the quark initiated channels are still unavailable they are not directly accessible from the $H+2 j$ amplitudes since they have been computed in the effective theory where the Higgs couples only to gluons. The necessary splitting amplitudes could be extracted from the vector boson plus four parton one-loop amplitudes [71].

Secondly when integrating the splitting functions over the unresolved phase space the expansion of the limit may be required to higher order in the dimensional regularisation parameter $\epsilon$. This would require a new computation of the one-loop matrix elements valid in $D=4-2 \epsilon$ dimensions which is quite feasible using modern unitarity methods. The appearance of the one-loop pentagon function in the full $D$-dimensional amplitude may complicate this part of the computation even if it is only required in the triple collinear limit. 
We hope that the expressions presented here will be of use in future high precision QCD computations.

\section{Acknowledgments}

We are grateful to Franz Herzog, Tom Melia and Einan Gardi for useful discussions. The work of S.B. is supported an STFC Rutherford Fellowship ST/L004925/1.

\section{A Generation of collinear phase space points}

In this appendix we illustrate a practical way to generate a set of on-shell $n$-particle phasespace points where the first $m$ particles approach the collinear limit $1\|\cdots\| m$. The limit is approached by varying a single free parameter $\delta$ as $\delta \rightarrow 0$ and it is based on the parametrisation presented in section 3. This has been used for the numerical checks we discussed in section 7 .

As a first step we generate an on-shell $(n-m+1)$-particle phase space point defining the set of momenta

$$
\left\{\tilde{P}, p_{m+1}(0), p_{m+2}(0), \ldots, p_{n}(0)\right\}
$$

where, as suggested by the notation, $p_{i}(0)$ for $i \geq m+1$ are the momenta of the noncollinear particles at $\delta=0$, while $\tilde{P}$ is the sum of the collinear momenta in the limit. We then define the exact collinear limit as the set of momenta

$$
\left\{z_{1} \tilde{P}, z_{2} \tilde{P}, \ldots, z_{m} \tilde{P}, p_{m+1}(0), \ldots, p_{n}(0)\right\}
$$

where $z_{i}$ are randomly generated real numbers satisfying eq. (3.6). In order to avoid regions with soft kinematics (which would introduce other kinds of singularities) one can generate a set of random numbers between, for example, 1 and 3 and divide them by their sum.

In order to define the orthogonal direction we must specify the reference vector $\eta$ appearing in eq. (3.2). A particular convenient choice is one of the non-collinear vectors, i.e.

$$
\eta^{\mu}=p_{m+1}^{\mu}(0)
$$

The orthogonal direction is thus spanned by the two complex vectors

$$
\frac{\left\langle\tilde{P}\left|\gamma^{\mu}\right| \eta\right]}{2}, \quad \frac{\left\langle\eta\left|\gamma^{\mu}\right| \tilde{P}\right]}{2} .
$$

While these are particularly convenient when working with the spinor-helicity formalism, for numerical checks with real kinematics it is convenient to define two real linear combinations

$$
v_{1, \perp}^{\mu}=\frac{1}{2}\left(\frac{\left\langle\tilde{P}\left|\gamma^{\mu}\right| \eta\right]}{2}+\frac{\left\langle\eta\left|\gamma^{\mu}\right| \tilde{P}\right]}{2}\right), \quad v_{2, \perp}^{\mu}=\frac{1}{2 i}\left(\frac{\left\langle\tilde{P}\left|\gamma^{\mu}\right| \eta\right]}{2}-\frac{\left\langle\eta\left|\gamma^{\mu}\right| \tilde{P}\right]}{2}\right) .
$$

Hence the orthogonal vectors $k_{T, i}^{\mu}$ are defined as

$$
k_{T, i}^{\mu}=y_{1, i} v_{1, \perp}^{\mu}+y_{2, i} v_{2, \perp}^{\mu}
$$


where $y_{1, i}$ and $y_{2, i}$ are randomly generated real numbers satisfying

$$
\sum_{i} y_{1, i}=\sum_{i} y_{2, i}=0
$$

The variables $y_{1, i}$ and $y_{2, i}$ are related to the spinor variables $\left\langle z_{i}\right\rangle,\left[z_{i}\right],\left\langle\omega_{i}\right\rangle$ and $\left[\omega_{i}\right]$ introduced in section 3 by

$$
y_{1, i}=\left\langle z_{i}\right\rangle\left[\omega_{i}\right]+\left\langle\omega_{i}\right\rangle\left[z_{i}\right], \quad y_{2, i}=i\left(\left\langle z_{i}\right\rangle\left[\omega_{i}\right]-\left\langle\omega_{i}\right\rangle\left[z_{i}\right]\right),
$$

as one can check by requiring consistency with eq. (3.9). As already stated, these spinor variables differ by a phase from the usual parametrisation in terms of $\sqrt{z_{i}}$. If

$$
\left\langle z_{i}\right\rangle=\left[z_{i}\right]^{*}=\sqrt{z_{i}} e^{i \theta}, \quad\left\langle\omega_{i}\right\rangle=\left[\omega_{i}\right]^{*}=\sqrt{\omega_{i}} e^{i \phi}
$$

then

$$
y_{1, i}=2 \sqrt{z_{i} \omega_{i}} \cos (\phi-\theta), \quad y_{2, i}=2 \sqrt{z_{i} \omega_{i}} \sin (\phi-\theta) .
$$

From here it is easy to see that while the parametrisation in terms of $\left\langle z_{i}\right\rangle$ and $\left[z_{i}\right]$ has the advantage of producing results that are analytic functions of the spinor variables in the complex plane, the parametrisation in terms of $\sqrt{z_{i}}$ is in fact entirely equivalent in the physical region.

Using $k_{T, i}^{\mu}$ as in eq. (A.6), one can simply define the momenta $p_{1}, \ldots, p_{m}$ for any value of the free parameter $\delta$ using eq. (3.2). With our choice of $\eta$ we can absorb the recoil by defining

$$
\begin{aligned}
p_{m+1}^{\mu}(\delta) & =\left(1+\sum_{i=0}^{m} \frac{\delta^{2} k_{T, i}^{2}}{2 z_{i}(\tilde{P} \cdot \eta)}\right) p_{m+1}^{\mu}(0) \\
p_{i}^{\mu}(\delta) & =p_{i}^{\mu}(0), \quad m+2 \leq i \leq n .
\end{aligned}
$$

Open Access. This article is distributed under the terms of the Creative Commons Attribution License (CC-BY 4.0), which permits any use, distribution and reproduction in any medium, provided the original author(s) and source are credited.

\section{References}

[1] S. Catani and M.H. Seymour, A General algorithm for calculating jet cross-sections in NLO QCD, Nucl. Phys. B 485 (1997) 291 [Erratum ibid. B 510 (1998) 503] [hep-ph/9605323] [INSPIRE].

[2] S. Frixione, Z. Kunszt and A. Signer, Three jet cross-sections to next-to-leading order, Nucl. Phys. B 467 (1996) 399 [hep-ph/9512328] [INSPIRE].

[3] A. Gehrmann-De Ridder, T. Gehrmann and E.W.N. Glover, Antenna subtraction at NNLO, JHEP 09 (2005) 056 [hep-ph/0505111] [INSPIRE].

[4] S. Catani and M. Grazzini, An NNLO subtraction formalism in hadron collisions and its application to Higgs boson production at the LHC, Phys. Rev. Lett. 98 (2007) 222002 [hep-ph/0703012] [INSPIRE]. 
[5] M. Czakon, A novel subtraction scheme for double-real radiation at NNLO, Phys. Lett. B 693 (2010) 259 [arXiv: 1005.0274] [INSPIRE].

[6] R. Boughezal, K. Melnikov and F. Petriello, A subtraction scheme for NNLO computations, Phys. Rev. D 85 (2012) 034025 [arXiv:1111.7041] [InSPIRE].

[7] M. Czakon and D. Heymes, Four-dimensional formulation of the sector-improved residue subtraction scheme, Nucl. Phys. B 890 (2014) 152 [arXiv:1408.2500] [INSPIRE].

[8] V. Del Duca, C. Duhr, G. Somogyi, F. Tramontano and Z. Trócsányi, Higgs boson decay into b-quarks at NNLO accuracy, JHEP 04 (2015) 036 [arXiv:1501.07226] [INSPIRE].

[9] S. Catani and M. Grazzini, Infrared factorization of tree level QCD amplitudes at the next-to-next-to-leading order and beyond, Nucl. Phys. B 570 (2000) 287 [hep-ph/9908523] [INSPIRE].

[10] Z. Bern, L.J. Dixon, D.C. Dunbar and D.A. Kosower, One loop n point gauge theory amplitudes, unitarity and collinear limits, Nucl. Phys. B 425 (1994) 217 [hep-ph/9403226] [INSPIRE].

[11] Z. Bern, L.J. Dixon and D.A. Kosower, One loop corrections to two quark three gluon amplitudes, Nucl. Phys. B 437 (1995) 259 [hep-ph/9409393] [InSPIRE].

[12] D.A. Kosower and P. Uwer, One loop splitting amplitudes in gauge theory, Nucl. Phys. B 563 (1999) 477 [hep-ph/9903515] [INSPIRE].

[13] Z. Bern, V. Del Duca and C.R. Schmidt, The Infrared behavior of one loop gluon amplitudes at next-to-next-to-leading order, Phys. Lett. B 445 (1998) 168 [hep-ph/9810409] [INSPIRE].

[14] Z. Bern, V. Del Duca, W.B. Kilgore and C.R. Schmidt, The infrared behavior of one loop QCD amplitudes at next-to-next-to leading order, Phys. Rev. D 60 (1999) 116001 [hep-ph/9903516] [INSPIRE].

[15] C. Anastasiou, C. Duhr, F. Dulat, F. Herzog and B. Mistlberger, Higgs Boson Gluon-Fusion Production in QCD at Three Loops, Phys. Rev .Lett. 114 (2015) 212001 [arXiv:1503.06056] [INSPIRE].

[16] C. Anastasiou et al., Higgs boson gluon-fusion production at threshold in $N^{3} L O Q C D$, Phys. Lett. B 737 (2014) 325 [arXiv:1403.4616] [INSPIRE].

[17] C. Anastasiou et al., Higgs boson gluon-fusion production beyond threshold in $N^{3} L O Q C D$, JHEP 03 (2015) 091 [arXiv: 1411.3584] [INSPIRE].

[18] Y. Li, A. von Manteuffel, R.M. Schabinger and H.X. Zhu, Soft-virtual corrections to Higgs production at $N^{3} L O$, Phys. Rev. D 91 (2015) 036008 [arXiv:1412.2771] [inSPIRE].

[19] P.A. Baikov, K.G. Chetyrkin, A.V. Smirnov, V.A. Smirnov and M. Steinhauser, Quark and gluon form factors to three loops, Phys. Rev. Lett. 102 (2009) 212002 [arXiv:0902.3519] [INSPIRE].

[20] T. Gehrmann, E.W.N. Glover, T. Huber, N. Ikizlerli and C. Studerus, Calculation of the quark and gluon form factors to three loops in QCD, JHEP 06 (2010) 094 [arXiv: 1004.3653] [INSPIRE].

[21] T. Gehrmann, E.W.N. Glover, T. Huber, N. Ikizlerli and C. Studerus, The quark and gluon form factors to three loops in QCD through to $O\left(\right.$ eps $\left.^{2}\right)$, JHEP 11 (2010) 102 [arXiv: 1010.4478] [INSPIRE]. 
[22] C. Anastasiou, C. Duhr, F. Dulat, F. Herzog and B. Mistlberger, Real-virtual contributions to the inclusive Higgs cross-section at $N^{3} L O$, JHEP 12 (2013) 088 [arXiv:1311.1425] [INSPIRE].

[23] W.B. Kilgore, One-loop single-real-emission contributions to $p p \rightarrow H+X$ at next-to-next-to-next-to-leading order, Phys. Rev. D 89 (2014) 073008 [arXiv:1312.1296] [INSPIRE].

[24] C. Duhr and T. Gehrmann, The two-loop soft current in dimensional regularization, Phys. Lett. B 727 (2013) 452 [arXiv: 1309.4393] [INSPIRE].

[25] Y. Li and H.X. Zhu, Single soft gluon emission at two loops, JHEP 11 (2013) 080 [arXiv: 1309.4391] [INSPIRE].

[26] C. Duhr, T. Gehrmann and M. Jaquier, Two-loop splitting amplitudes and the single-real contribution to inclusive Higgs production at $N^{3}$ LO, JHEP 02 (2015) 077 [arXiv:1411.3587] [INSPIRE].

[27] F. Dulat and B. Mistlberger, Real-Virtual-Virtual contributions to the inclusive Higgs cross section at N3LO, arXiv:1411.3586 [INSPIRE].

[28] Y. Li, A. von Manteuffel, R.M. Schabinger and H.X. Zhu, $N^{3} L O$ Higgs boson and Drell-Yan production at threshold: The one-loop two-emission contribution, Phys. Rev. D 90 (2014) 053006 [arXiv: 1404.5839] [INSPIRE].

[29] C. Anastasiou et al., Soft Expansion of Double-Real-Virtual Corrections to Higgs Production at $N^{3} L O$, arXiv: 1505.04110 [INSPIRE].

[30] C. Anastasiou, C. Duhr, F. Dulat and B. Mistlberger, Soft triple-real radiation for Higgs production at N3LO, JHEP 07 (2013) 003 [arXiv: 1302.4379] [INSPIRE].

[31] O.V. Tarasov, A.A. Vladimirov and A. Yu. Zharkov, The Gell-Mann-Low Function of QCD in the Three Loop Approximation, Phys. Lett. B 93 (1980) 429 [INSPIRE].

[32] S.A. Larin and J.A.M. Vermaseren, The Three loop QCD $\beta$-function and anomalous dimensions, Phys. Lett. B 303 (1993) 334 [hep-ph/9302208] [INSPIRE].

[33] T. van Ritbergen, J.A.M. Vermaseren and S.A. Larin, The Four loop $\beta$-function in quantum chromodynamics, Phys. Lett. B 400 (1997) 379 [hep-ph/9701390] [INSPIRE].

[34] M. Czakon, The Four-loop QCD $\beta$-function and anomalous dimensions, Nucl. Phys. B 710 (2005) 485 [hep-ph/0411261] [INSPIRE].

[35] A. Vogt, S. Moch and J.A.M. Vermaseren, The Three-loop splitting functions in QCD: The Singlet case, Nucl. Phys. B 691 (2004) 129 [hep-ph/0404111] [InSPIRE].

[36] S. Moch, J.A.M. Vermaseren and A. Vogt, The Three loop splitting functions in QCD: The Nonsinglet case, Nucl. Phys. B 688 (2004) 101 [hep-ph/0403192] [INSPIRE].

[37] C. Anastasiou, S. Buehler, C. Duhr and F. Herzog, NNLO phase space master integrals for two-to-one inclusive cross sections in dimensional regularization, JHEP 11 (2012) 062 [arXiv: 1208.3130] [INSPIRE].

[38] M. Höschele, J. Hoff, A. Pak, M. Steinhauser and T. Ueda, Higgs boson production at the LHC: NNLO partonic cross sections through order $\epsilon$ and convolutions with splitting functions to $N^{3}$ LO, Phys. Lett. B 721 (2013) 244 [arXiv:1211.6559] [INSPIRE].

[39] S. Buehler and A. Lazopoulos, Scale dependence and collinear subtraction terms for Higgs production in gluon fusion at N3LO, JHEP 10 (2013) 096 [arXiv:1306.2223] [INSPIRE]. 
[40] D.A. Kosower, All order collinear behavior in gauge theories, Nucl. Phys. B 552 (1999) 319 [hep-ph/9901201] [INSPIRE].

[41] Z. Bern, L.J. Dixon and D.A. Kosower, Two-loop $g \rightarrow$ g splitting amplitudes in QCD, JHEP 08 (2004) 012 [hep-ph/0404293] [INSPIRE].

[42] S.D. Badger and E.W.N. Glover, Two loop splitting functions in QCD, JHEP 07 (2004) 040 [hep-ph/0405236] [INSPIRE].

[43] J.M. Campbell and E.W.N. Glover, Double unresolved approximations to multiparton scattering amplitudes, Nucl. Phys. B 527 (1998) 264 [hep-ph/9710255] [INSPIRE].

[44] V. Del Duca, A. Frizzo and F. Maltoni, Factorization of tree QCD amplitudes in the high-energy limit and in the collinear limit, Nucl. Phys. B 568 (2000) 211 [hep-ph/9909464] [INSPIRE].

[45] T.G. Birthwright, E.W.N. Glover, V.V. Khoze and P. Marquard, Multi-gluon collinear limits from MHV diagrams, JHEP 05 (2005) 013 [hep-ph/0503063] [INSPIRE].

[46] T.G. Birthwright, E.W.N. Glover, V.V. Khoze and P. Marquard, Collinear limits in QCD from MHV rules, JHEP 07 (2005) 068 [hep-ph/0505219] [INSPIRE].

[47] S. Catani, D. de Florian and G. Rodrigo, The Triple collinear limit of one loop QCD amplitudes, Phys. Lett. B 586 (2004) 323 [hep-ph/0312067] [INSPIRE].

[48] G.F.R. Sborlini, D. de Florian and G. Rodrigo, Triple collinear splitting functions at NLO for scattering processes with photons, JHEP 10 (2014) 161 [arXiv:1408.4821] [INSPIRE].

[49] G.F.R. Sborlini, D. de Florian and G. Rodrigo, Polarized triple-collinear splitting functions at NLO for processes with photons, JHEP 03 (2015) 021 [arXiv:1409.6137] [INSPIRE].

[50] C.F. Berger, V. Del Duca and L.J. Dixon, Recursive Construction of Higgs-Plus-Multiparton Loop Amplitudes: The Last of the Phi-nite Loop Amplitudes, Phys. Rev. D 74 (2006) 094021 [Erratum ibid. D 76 (2007) 099901] [hep-ph/0608180] [INSPIRE].

[51] S.D. Badger and E.W.N. Glover, One-loop helicity amplitudes for $H \rightarrow$ gluons: The All-minus configuration, Nucl. Phys. Proc. Suppl. 160 (2006) 71 [hep-ph/0607139] [INSPIRE].

[52] S.D. Badger, E.W.N. Glover and K. Risager, One-loop phi-MHV amplitudes using the unitarity bootstrap, JHEP 07 (2007) 066 [arXiv:0704.3914] [INSPIRE].

[53] E.W.N. Glover, P. Mastrolia and C. Williams, One-loop phi-MHV amplitudes using the unitarity bootstrap: The General helicity case, JHEP 08 (2008) 017 [arXiv:0804.4149] [INSPIRE].

[54] S. Badger, E.W. Nigel Glover, P. Mastrolia and C. Williams, One-loop Higgs plus four gluon amplitudes: Full analytic results, JHEP 01 (2010) 036 [arXiv:0909.4475] [INSPIRE].

[55] L.J. Dixon and Y. Sofianatos, Analytic one-loop amplitudes for a Higgs boson plus four partons, JHEP 08 (2009) 058 [arXiv:0906.0008] [INSPIRE].

[56] S. Badger, J.M. Campbell, R.K. Ellis and C. Williams, Analytic results for the one-loop NMHV Hqqgg amplitude, JHEP 12 (2009) 035 [arXiv:0910.4481] [INSPIRE].

[57] D.A. Kosower, Antenna factorization of gauge theory amplitudes, Phys. Rev. D 57 (1998) $5410[\mathrm{hep}-\mathrm{ph} / 9710213]$ [INSPIRE].

[58] C. Duhr, Mathematical aspects of scattering amplitudes, arXiv:1411.7538 [INSPIRE]. 
[59] H.J. Lu and C.A. Perez, Massless one loop scalar three point integral and associated Clausen, Glaisher and L functions, SLAC-PUB-5809 (1992).

[60] Z. Bern, L.J. Dixon and D.A. Kosower, Dimensionally regulated pentagon integrals, Nucl. Phys. B 412 (1994) 751 [hep-ph/9306240] [INSPIRE].

[61] T. Binoth, J.P. Guillet, G. Heinrich and C. Schubert, Calculation of one loop hexagon amplitudes in the Yukawa model, Nucl. Phys. B 615 (2001) 385 [hep-ph/0106243] [INSPIRE].

[62] A. van Hameren, J. Vollinga and S. Weinzierl, Automated computation of one-loop integrals in massless theories, Eur. Phys. J. C 41 (2005) 361 [hep-ph/0502165] [INSPIRE].

[63] R.K. Ellis and G. Zanderighi, Scalar one-loop integrals for QCD, JHEP 02 (2008) 002 [arXiv:0712.1851] [INSPIRE].

[64] D. Forde, Direct extraction of one-loop integral coefficients, Phys. Rev. D 75 (2007) 125019 [arXiv: 0704.1835] [INSPIRE].

[65] R. Kleiss and H. Kuijf, Multi-gluon cross-sections and five jet production at hadron colliders, Nucl. Phys. B 312 (1989) 616 [inSPIRE].

[66] V. Del Duca, L.J. Dixon and F. Maltoni, New color decompositions for gauge amplitudes at tree and loop level, Nucl. Phys. B 571 (2000) 51 [hep-ph/9910563] [INSPIRE].

[67] M.L. Mangano, S.J. Parke and Z. Xu, Duality and multi-gluon scattering, Nucl. Phys. B 298 (1988) 653 [INSPIRE].

[68] M.L. Mangano and S.J. Parke, Multiparton amplitudes in gauge theories, Phys. Rept. 200 (1991) 301 [hep-th/0509223] [INSPIRE].

[69] S. Badger, B. Biedermann, P. Uwer and V. Yundin, Numerical evaluation of virtual corrections to multi-jet production in massless QCD, Comput. Phys. Commun. 184 (2013) 1981 [arXiv: 1209.0100] [INSPIRE].

[70] A. van Hameren, OneLOop: For the evaluation of one-loop scalar functions, Comput. Phys. Commun. 182 (2011) 2427 [arXiv: 1007.4716] [INSPIRE].

[71] Z. Bern, L.J. Dixon and D.A. Kosower, One loop amplitudes for $e^{+} e^{-}$to four partons, Nucl. Phys. B 513 (1998) 3 [hep-ph/9708239] [INSPIRE]. 